\title{
A Robust Optimization Approach for Optimal Power Flow Solutions Using Rao Algorithms
}

\author{
Saket Gupta ${ }^{1}$, Narendra Kumar ${ }^{1}$, Laxmi Srivastava ${ }^{2} \oplus$, Hasmat Malik ${ }^{3, *} \mathbb{D}$, Amjad Anvari-Moghaddam ${ }^{4, *}$ \\ and Fausto Pedro García Márquez ${ }^{5}$ (D) \\ 1 Electrical Engineering Department, Delhi Technological University, Delhi 110042, India; \\ sguptamits@gmail.com (S.G.); dnk_1963@yahoo.com (N.K.) \\ 2 Electrical Engineering Department, Madhav Institute of Technology \& Science, Gwalior 474005, India; \\ srivastaval@hotmail.com \\ 3 Berkeley Education Alliance for Research in Singapore (BEARS), University Town, NUS Campus, \\ Singapore 138602, Singapore \\ 4 Department of Energy (AAU Energy), Aalborg University, 9220 Aalborg, Denmark \\ 5 Ingenium Research Group, Universidad Castilla-La Mancha, 13071 Ciudad Real, Spain; \\ FaustoPedro.Garcia@uclm.es \\ * Correspondence: hasmat.malik@gmail.com (H.M.); aam@energy.aau.dk (A.A.-M.)
}

Citation: Gupta, S.; Kumar, N.;

Srivastava, L.; Malik, H.;

Anvari-Moghaddam, A.;

García Márquez, F.P. A Robust

Optimization Approach for Optimal

Power Flow Solutions Using Rao

Algorithms. Energies 2021, 14, 5449.

https://doi.org/10.3390/en14175449

Academic Editor: Jarosław Konieczny

Received: 11 June 2021

Accepted: 26 August 2021

Published: 1 September 2021

Publisher's Note: MDPI stays neutral with regard to jurisdictional claims in published maps and institutional affiliations.

\begin{abstract}
This paper offers three easy-to-use metaphor-less optimization algorithms proposed by Rao to solve the optimal power flow (OPF) problem. Rao algorithms are parameter-less optimization algorithms. As a result, algorithm-specific parameter tuning is not required at all. This quality makes these algorithms simple to use and able to solve various kinds of complex constrained optimization and engineering problems. In this paper, the main aim to solve the OPF problem is to find the optimal values of the control variables in a given electrical network for fuel cost minimization, real power losses minimization, emission cost minimization, voltage profile improvement, and voltage stability enhancement, while all the operating constraints are satisfied. To demonstrate the efficacy of Rao algorithms, these algorithms have been employed in three standard IEEE test systems (30-bus, 57-bus, and 118-bus) to solve the OPF problem. The OPF results of Rao algorithms and the results provided by other swarm intelligence (SI)/evolutionary computing (EC)-based algorithms published in recent literature have been compared. Based on the outcomes, Rao algorithms are found to be robust and superior to their competitors.
\end{abstract}

Keywords: fuel cost; optimal power flow; power losses; voltage stability; voltage profile; emission

\section{Introduction}

With the inclusion of different kinds of power electronic appliances and renewable energy sources in modern inter-connected restructured power systems, the importance of solving the OPF problem is increasing day by day. Optimal power flow results are needed for economic operation, planning, and control of the existing electrical grid and future expansion planning. In a given electrical network, the OPF solution must regulate the control or decision variables set in a feasible region that optimizes pre-defined objective functions. In the formulation of the OPF problem, fuel cost minimization (FCM) is frequently used as a primary objective function in addition to other objectives like voltage stability enhancement (VSE), voltage profile improvement (VPI), real power losses minimization (RPLM), and emission cost minimization (ECM) via readjustment of control variables, taking into account both operational and physical constraints [1]. The mathematical formulation of the OPF problem is complex. It is a highly non-linear, high-dimensional, non-differential, multi-modal, and non-convex problem with discrete and continuous control variables. Carpentier introduced a first-time OPF problem based on the economic load dispatch in the early 1960 s $[2,3]$. 
Many classical optimization techniques (COTs) [4,5] such as linear programming, non-linear programming, mixed integer programming, interior point method, etc., were employed during the early decades to handle OPF problems. While some COTs have outstanding convergence properties, many of them are often used in the industry. Their disadvantages include the following: most of the conventional algorithms are deterministic, except hill-climbing, thus they are unable to find the global optimal solution, and they cannot efficiently manage binary or integer variables. In addition, conventional optimization methods require initial points (except for linear programming and convex optimization), thus the COTs are not appropriate for solving the OPF problem.

Various evolutionary computing-based algorithms were proposed in recent literature to overcome the demerits of classical optimization techniques and solve the complex constrained optimization problem. In a couple of years, the EC techniques are attracting power engineers and researchers to solve OPF problems because of their efficacy in handling various types of engineering and complex optimization problems [6,7]. Some of these techniques are the: bat search algorithm (BSA) [8]; improved colliding bodies optimization (ICBO) [9]; back-hole-based optimization (BHBO) [10]; multi-agent-based differential evolution approach (MADE) [11]; adaptive group search optimization (AGSO) [12]; biogeography-based optimization (BBO) [13]; moth swarm algorithm (MSA) [14]; water evaporation algorithm (WEA) [15]; symbiotic organisms search algorithm (SOS) [16]; treeseed algorithm (TS) [17]; differential search algorithm (DSA) [18]; salp swarm optimizer algorithm (SSO) [19], etc.

Rao algorithms and many modified versions of Rao algorithms have been used to solve a wide range of optimization problems including the optimal reactive power dispatch with renewable energy [20], multi-objective optimization of selected thermodynamic cycles [21], constrained design optimization of selected mechanical system components [22], engineering design optimization [23], estimation of photovoltaic cell parameters [24], classification of Parkinson's disease [25], OPF problem with renewable energy sources [26], multi-objective design optimization of selected heat sinks [27], and many more.

The authors found that all EC/SI-based algorithms have some advantages and disadvantages through the literature survey. Two main parts of any EC/SI-based algorithm are exploration and exploitation, or intensification and diversification. Some algorithms have good exploration capability but poor exploitation, and vice versa. Some algorithms are more suitable to solve certain types of problems than others. It is logically proved that any single EC/SI-based optimization algorithm does not have the potential to solve various types of engineering and complex optimization problems, thus, the "No Free Lunch" theorem encourages the development of new algorithms [28].

Meta-heuristic algorithms, notwithstanding their benefits, have several drawbacks. They need parameter tuning to find the near-global best solution. It has been observed that parameter tuning of meta-heuristic optimization algorithms plays a very important role and is a very crucial and time-costly task for solving a given optimization problem. Therefore in this paper, the authors proposed a robust optimization technique called Rao algorithms to resolve the OPF problem. The proposed algorithms are comparatively new meta-heuristic optimization algorithms developed by Rao [29,30].

Rao algorithms are parameter-less optimization algorithms. As a result, algorithmspecific parameter tuning is not required at all. This quality makes these algorithms simple to apply for solving different kinds of optimization problems. The working principle of Rao algorithms is based on the worst and best solutions achieved throughout the optimization and random interaction between the candidate solutions.

The main contributions of this paper are:

- To develop Rao algorithms to solve OPF problems with six objective functions, namely FCM, VSE under normal and contingency conditions, VPI, RPLM, and ECM.

- To apply Rao algorithms to solve various multi-objective OPF problems by transforming the multi-objective OPF problem into a single objective OPF problem using weighing factors. 
- To check the efficiency and supremacy of Rao algorithms by applying these algorithms to solve the OPF problem in three standard IEEE (30-bus, 57-bus, and 118-bus) test systems.

- To compare the simulation outcomes acquired by Rao algorithms for the abovementioned objective functions with the results of other methods mentioned in recent literature.

- The OPF results demonstrate that the suggested Rao algorithms are efficient and robust in most of the cases over other popular methods, which are reported in recent literature.

In this paper, the authors propose Rao algorithms, which are recently devolved powerful optimization algorithms, to solve OPF problems considering technical and economical objective functions in three standard IEEE (30-bus, 57-bus, and 118-bus) systems. The rest of the paper is organized as follows: Section 2 covers the representation of OPF problems; Section 3 provides details of Rao algorithms to solve OPF problems; Section 4 provides the OPF results attained by Rao algorithms; and in Section 6, the conclusion is provided.

\section{Problem Formulation}

The OPF problem formulation aims to find the optimal setting of the control variables for a given electrical network that optimizes the pre-defined objective functions while all operating constraints and power flows are within the operating limit [10,11]. Mathematically, the objective function, together with the operating constraints of the OPF problem selected in this work, is given by Equations (1)-(3):

$$
\text { Minimize } Z_{\min }(m, n)
$$

Subject to the constraints;

$$
g(m, n)=0
$$

and

$$
h(m, n) \leq 0(3)
$$

$Z_{\min }(m, n)$ is an objective function that needs to be optimized; $g$ is the equality constraints representing the non-linear load flow equations; and $h$ is the inequality constraints representing the system operating limits.

The dependent variables $(m)$ and control variables $(n)$ are described in Equations (4) and (5):

$$
\begin{gathered}
m=\left[P_{g_{1}}, V_{1} \ldots V_{N L B}, Q_{g 1}, \ldots Q_{g N G N}, S_{1}, \ldots S_{N t t}\right] \\
n=\left[P_{g_{2}} \ldots P_{g_{N G N}}, V_{g_{1}} \ldots V_{g_{N G N}}, Q_{C_{1}} \ldots Q_{C_{N C}}, \ldots T_{1} \ldots T_{N T R}\right]
\end{gathered}
$$

where, $P_{g}$ and $Q_{g}$ denote the active and reactive power outputs of generator units. $V_{L}$ and $V_{g}$ represent the load and generator bus voltages, respectively. $Q_{C}$ and $T_{T R}$ represent the shunt VAR compensation and transformer tap-setting, respectively. $P_{g_{1}}$ is the slack bus active power output. $N G N, N C, N T R, N L B$, and $N t l$ correspond to the number of generators, number of VAR compensation units, numbers of regulating transformers, number of load buses, and number of transmission lines, respectively.

\section{Constraints}

Two types of constraints are involved in the OPF problem, namely equality and inequality [31].

\section{(a) Equality Constraints}

The equality constraints $g(m, n)$ represent the load flow equation and can be described by Equations (6) and (7):

$$
\begin{gathered}
P_{\text {Loss }}=\sum_{i=1}^{N B} P_{g i}-\sum_{i=1}^{N B} P_{d i} \\
Q_{\text {Loss }}=\sum_{i=1}^{N B} Q_{g i}-\sum_{i=1}^{N B} Q_{d i}
\end{gathered}
$$


where $P_{\text {Loss }}$ and $Q_{\text {Loss }}$ denote the total active and reactive power loss for a given electrical system, respectively. $N B$ represents the number of buses. $P_{d i}$ and $Q_{d i}$ are the active and reactive power demands at the $i$ th bus.

\section{(b) Inequality Constraints}

The operational limits of various power system equipment are expressed by the inequality constraints $h(m, n)$, which are described by Equations (8)-(14):

- Generator Constraints:

For each generator unit, the active powers $\left(P_{g k}\right)$, reactive powers $\left(Q_{g k}\right)$, and bus voltage $\left(V_{g k}\right)$ should lie in between their minimum and maximum limits.

$$
\begin{gathered}
P_{g_{k}}^{\min } \leq P_{g_{k}} \leq P_{g_{k}}^{\max } k=1 \ldots \ldots N G N \\
V_{g_{k}}^{\text {min }} \leq V_{g_{k}} \leq V_{g_{k}}^{\text {max }} k=1 \ldots \ldots N G N \\
Q_{g_{k}}^{\min } \leq Q_{g_{k}} \leq Q_{g_{k}}^{\max } k=1 \ldots \ldots N G N
\end{gathered}
$$

- $\quad$ Shunt VAR compensator constraints:

The controllable VAR sources $\left(Q_{C_{k}}\right)$ are regulated to their lowest and maximum limits:

$$
Q_{C_{k}}^{\min } \leq Q_{C_{k}} \leq Q_{C_{k}}^{\max } k=1 \ldots N C
$$

- Transformer Constraints:

Transformer tap settings $\left(T_{k}\right)$ are retained within the maximum and minimum limits.

$$
T_{k}^{\min } \leq T_{k} \leq T_{k}^{\max } k=1 \ldots \ldots \text { NTR }
$$

- Security Constraints:

The voltage at load buses $\left(V_{L_{k}}\right)$ and the power flow in transmission lines $\left(S_{l_{k}}\right)$ should be retained within their respective minimum and maximum limits.

$$
\begin{gathered}
V_{L_{k}}^{\min } \leq V_{L_{k}} \leq V_{L_{k}}^{\max } k=1 \ldots \ldots N B \\
S_{l_{k}} \leq S_{l_{k}}^{\max } k=1 \ldots \ldots t l
\end{gathered}
$$

\section{(c) Incorporation of Constraints}

The penalty factor approach is used in the OPF problem to efficiently include the working limits of operating constraints, such as bus voltage constraints, line flows, and generator constraints. The penalty factor approach penalizes each violation by multiplying it by a large number so that infeasible solutions are rejected and only feasible solutions are considered. To find feasible solutions [30], the above-mentioned inequality constraints are included and the augmented objective function is obtained by Equation (15).

$$
\begin{gathered}
Z_{\text {aug }}=Z_{\text {min }}(.)+C_{1} \cdot H\left(P_{g_{1}}\right)+C_{2} \sum_{i=1}^{N G N} H\left(Q_{g_{i}}\right)+C_{3} \sum_{i=1}^{N L B} H\left(V_{L_{i}}\right)+C_{4} \sum_{i=1}^{N t l} H\left(S_{l_{i}}\right) \\
H\left(x_{i}\right)=\left\{\begin{array}{l}
\left(x_{i}-x_{i, \max }\right)^{2} \text { if } x_{i}>x_{i, \max } \\
\left(x_{i, \min }-x_{i}\right)^{2} \text { if } x_{i}<x_{i, \min } \\
0 \text { if } x_{i, \min } \leq x_{i} \leq x_{i, \max }
\end{array}\right.
\end{gathered}
$$

where $H\left(x_{i}\right)$ is the penalty function of variable $x_{i}$. Here, $x_{i}$ shows a dependent variable. In addition, $x_{i, \max }$, and $x_{i, \min }$ are the upper and lower limits of the variable $x_{i}$, respectively.

The three variants of the proposed Rao algorithms are applied to solve the OPF problems considering inherent complexities such as non-linear, multi-extremism, high dimensionality, and non-convexity property. In this paper, all the control variables are considered to be continuous. 


\section{Rao Algorithms}

The proposed Rao algorithms employ the worst and best solutions, which can be obtained during the phases of optimization and random interaction among candidate solutions. The key benefit of the proposed algorithms is their algorithm-specific parameterless property and hence these algorithms can be easily implemented for solving different types of optimization problems [21-23,29].

Assume that there is an ' $m$ ' population size (i.e., candidate solutions, $k=1,2, \ldots$, $m$ ) and ' $n$ ' design variables (i.e., $j=1,2,3, \ldots, n$ ) for any iteration $i$. The best candidate will provide the best value of an objective function in all the candidate solutions, while the worst candidate will give the worst value of the objective function. During the $i$ th iteration, if $R_{i, j, k}$ is the $j$ th variable value for the $k$ th candidate, its value is updated according to Equations (17)-(19).

$$
\begin{gathered}
R_{j, k, i}{ }^{\prime}=R_{j, k, i}+\alpha_{1, j, i}\left(R_{j, b e s t, i}-R_{j, \text { wors }, i}\right) \\
R_{j, k, i}{ }^{\prime}=R_{j, k, i}+\alpha_{1, j, i}\left(R_{j, \text { best }, i}-R_{j, w o r s t, i}\right)+\alpha_{2, j, i}\left(\mid R_{j, k, i} \text { or } R_{j, l, i}|-| R_{j, l, i} \text { or } R_{j, k, i} \mid\right) \\
R_{j, k, i}{ }^{\prime}=R_{j, k, i}+\alpha_{1, j, i}\left(R_{j, b e s t, i}-\left|R_{j, w o r s t, i}\right|\right)+\alpha_{2, j, i}\left(\mid R_{j, k, i} \text { or } R_{j, l, i} \mid-\left(R_{j, l, i} \text { or } R_{j, k, i}\right)\right)
\end{gathered}
$$

In Equations (17)-(19), the value of the $j$ th variable for the best candidate is $R_{j, b e s t, i}$ and the value of the $j$ th variable for the worst candidate is $R_{j, w o r s t, i}$. The modified value of $R_{j, k, i}$ is $R_{j, k, i}{ }^{\prime}$. For the $j$ th variable, $\alpha_{1, j, i}$ and $\alpha_{2, j, i}$ are the two random numbers in the range $(0,1)$ during the $i$ th iteration [29].

The term " $R_{j, k, i}$ or $R_{j, l, i}$ " in Equations (17)-(19) shows that the solution for candidate $k$ is compared with any randomly picked candidate solution $l$ and the information is shared based on objective function values. If the $k$ th solution's objective function value is better than the $l$ th solution's objective function value, the term " $R_{j, k, i}$ or $R_{j, l, i}$ " becomes $R_{j, k, i}$ and in that case, " $R_{j, l, i}$ or $R_{j, k, i}$ " becomes $R_{j, l, i}$. Similarly, the term " $R_{j, k, i}$ or $R_{j, l, i}$ " becomes $R_{j, l, i}$ when the objective function value of the $l$ th solution is better than the fitness value of $k$ th solution and in such condition, the expression " $R_{j, l, i}$ or $R_{j, k, i}$ " becomes $R_{j, k, i}$.

The flowchart of the Rao algorithm is shown in Figure 1. The flowcharts for the Rao2 and Rao-3 algorithms will be the same, except that Equation (17) will be replaced by Equations (18) and (19) in either chart, respectively.

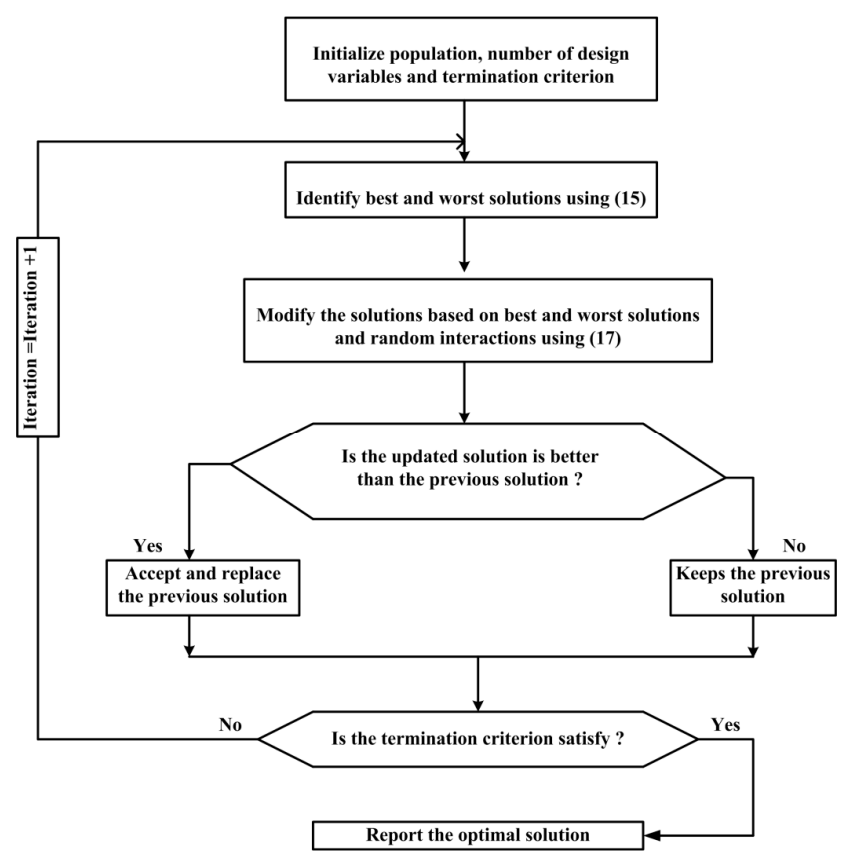

Figure 1. Flowchart of Rao-1 algorithm. Adapted from ref. [29]. 


\section{Computational Steps of Rao Algorithms for the OPF Problem}

The following are the computational steps for applying Rao algorithms:

1. Randomly generate the initial population with control variables and set the stopping criteria, i.e., It_max.

2. Set iterations count to $I t=0$.

3. Identify the worst and best solutions in the population by observing the value of the augmented objective function (15).

4. Update the solutions based on the worst and best solutions (17).

5. Proceed to step 6 if the updated solution is better than the previous solution; otherwise, proceed to step 7.

6. Replace the old solution with the new one. Go to step 8 .

7. Keep the old solution.

8. If $I t<I t \_m a x$, increase the count of iteration (i.e., $I t=I t+1$ ) by 1 and go to step 3 . Otherwise go to step 9.

9. Stop and display objective function value of best results.

\section{OPF Results and Discussion}

Three standard test systems, IEEE (30-, 57-, and 118-bus), are used to check the efficacy of the Rao algorithms considering various objective functions. Several trials have been carried out but the best results obtained and presented in this paper are with the population size (pop.) $=40$ and the maximum number of iterations (It_max) $=100$ for the IEEE 30-bus system, while pop. $=50$ and It_max $=150$ for the IEEE 57-bus and IEEE 118-bus systems. All the computations were carried out on a $1.7 \mathrm{GHz}$ Intel Processor, 4GB RAM, Core i3, and 64-bit operating system using the MATLAB-13a computing environment on a personal computer (PC).

In various cases of these systems, the converged solution strictly followed all the lower and upper operating limits, which included reactive and active power generation output, line loading, and load bus voltage magnitudes. Under this condition, the value of the penalty was observed to be zero, which guarantees a feasible solution. It has been seen that Rao algorithms' numerical results are better in most of the cases than the reported results as mentioned in recent literature.

Performance of Rao-1, Rao-2, and Rao-3 is found to be competitive with one another as mentioned in [30]. In this paper, different topologies have been considered because the different topologies have different problem dimensions and complexities. The proposed Rao algorithms have been applied on three standard IEEE test systems, i.e., 30-bus, 57-bus, and 118-bus, which have 24 control variables, 33 control variables, and 130 control variables, respectively, to test the efficacy of the proposed algorithm for different problems dimensions.

\subsection{IEEE 30-Bus Test System}

The system data along with control variables' operating limits are taken from Reference [32] and given in Table 1. The emission and fuel cost coefficients of the IEEE 30-bus system are taken from [33]. For this system, 30 runs were performed using Rao algorithms to solve the different objective functions of the OPF problem and the best results out of 30 independent trials are given in this paper.

Table 1. Details of the IEEE 30-bus test system.

\begin{tabular}{|c|c|c|c|c|c|c|c|c|}
\hline $\begin{array}{l}\text { Control } \\
\text { Variables }\end{array}$ & Nbus & $N t l$ & $N G N$ & $N L B$ & $N C$ & NTR & $\begin{array}{l}\text { Voltage Limit } \\
\text { (PQ Bus) }\end{array}$ & $\begin{array}{l}\text { Voltage Limit } \\
\text { (PV Bus) }\end{array}$ \\
\hline $\begin{array}{c}24 \\
(05+06+09+04)\end{array}$ & 30 & 41 & $\begin{array}{c}6\left(@ \mathrm{G}_{1}, \mathrm{G}_{2},\right. \\
\mathrm{G}_{5}, \mathrm{G}_{8}, \mathrm{G}_{11}, \\
\left.\mathrm{G}_{13}\right)\end{array}$ & 24 & $\begin{array}{c}9 \text { (@ Sh } 10, \text { Sh } 12, \text { Sh } \\
15, \text { Sh } 17, \text { Sh } 20, \text { Sh } 21, \\
\text { Sh } 23, \text { Sh } 24, \text { Sh } 29)\end{array}$ & $\begin{array}{c}4 \text { (@ Nt 11, } \\
\text { Nt 12, Nt } \\
15 \text { \& Nt 36) }\end{array}$ & $(0.94: 1.06)$ & $(0.95: 1.1)$ \\
\hline
\end{tabular}




\subsubsection{Case 1: Fuel Cost Minimization (FCM)}

Here, the first objective of the OPF problem is to reduce the total cost of generation or the fuel cost [8]. This function can be described as Equation (20):

$$
F_{\text {cost }}(a, b)=\sum_{i=1}^{N G N} f_{i}\left(P_{g_{i}}\right)(\$ / h)
$$

All the generating units' cost characteristics are quadratic and can be stated as Equation (21).

$$
f_{i}\left(P_{g_{i}}\right)=A_{i}+B_{i} P_{g_{i}}+C_{i} P_{g_{i}}^{2}(\$ / h)
$$

The minimum cost attained by the Rao-3 algorithm is $799.9683 \$ / \mathrm{h}$, while Rao-2 and Rao-1 algorithms provided the minimum fuel cost of $799.9918 \$ / \mathrm{h}$ and $800.4391 \mathrm{\$} / \mathrm{h}$, respectively. Table 2 compares the simulation results of Case 1 obtained by the proposed algorithms and other reported algorithms listed in recent literature. The OPF results of the proposed Rao-3 algorithm and optimal control variable settings are presented in Table 3. Based on the outcomes, it is clear that the Rao-3 algorithm provided the least value of the fuel cost as compared to the other methods. This demonstrates the effectiveness of the proposed Rao-3 algorithm as compared to Rao-2 and Rao-1 algorithms, as well as other competitors for this case. The fuel cost convergence characteristics of Case 1 are presented in Figure 2.

\begin{tabular}{|c|c|c|}
\hline Algorithm & Fuel Cost (\$/h) & Time (s) \\
\hline Rao-3 & 799.9683 & 89.56 \\
\hline Rao-2 & 799.9918 & 89.74 \\
\hline Rao-1 & 800.4391 & 91.62 \\
\hline MSA [14] & 800.5099 & - \\
\hline MPSO [14] & 800.5164 & - \\
\hline MDE [14] & 800.8399 & - \\
\hline MFO [14] & 800.6863 & - \\
\hline FPA [14] & 802.7983 & - \\
\hline DSA [18] & 800.3887 & - \\
\hline EEA [34] & 800.0831 & . \\
\hline Jaya [35] & 800.479 & - \\
\hline QOJA [36] & 800.352 & - \\
\hline MSO [37] & 801.571 & - \\
\hline IMFO [38] & 800.3848 & - \\
\hline MFO [38] & 800.6206 & - \\
\hline GA [38] & 800.4346 & - \\
\hline PSO [38] & 800.4075 & - \\
\hline TLBO [38] & 800.4104 & - \\
\hline ARCBBO [39] & 800.5159 & - \\
\hline RCBBO [39] & 800.8703 & - \\
\hline GWO [40] & 801.41 & - \\
\hline $\mathrm{DE}[40]$ & 801.23 & - \\
\hline
\end{tabular}

Table 2. Case 1: FCM in the IEEE 30-bus system. 
Table 2. Cont.

\begin{tabular}{ccc}
\hline Algorithm & Fuel Cost (\$/h) & Time (s) \\
\hline MGBICA [41] & 801.1409 & - \\
\hline GBICA [41] & 801.1513 & - \\
\hline ABC [42] & 800.66 & - \\
\hline SKH [43] & 800.5141 & - \\
\hline ECHT-DE [44] & 800.4148 & - \\
\hline SF-DE [44] & 800.4131 & - \\
\hline SP-DE [44] & 800.4293 .1 \\
\hline MGOA [45] & 800.4744 & - \\
\hline Tabu Search [46] & 800.29 & - \\
\hline
\end{tabular}

Table 3. Optimum values of control variables for Case 1, Case 2, and Case 3 of the IEEE 30-bus system.

\begin{tabular}{|c|c|c|c|c|c|c|c|c|c|c|}
\hline \multirow{2}{*}{$\begin{array}{c}\text { S. } \\
\text { Number }\end{array}$} & \multirow{2}{*}{$\begin{array}{c}\text { Control } \\
\text { Variable }\end{array}$} & \multicolumn{3}{|c|}{$\begin{array}{l}\text { Case } 1 \\
\text { (FCM) }\end{array}$} & \multicolumn{3}{|c|}{$\begin{array}{c}\text { Case } 2 \\
\text { (VPI) }\end{array}$} & \multicolumn{3}{|c|}{$\begin{array}{l}\text { Case } 3 \\
\text { (VSE) }\end{array}$} \\
\hline & & Rao-1 & Rao-2 & Rao-3 & Rao-1 & Rao-2 & Rao-3 & Rao-1 & Rao-2 & Rao-3 \\
\hline \multicolumn{11}{|c|}{ Generator active power output } \\
\hline 1 & $P_{\mathrm{G} 2}$ & 0.4869 & 0.4923 & 0.4879 & 0.4957 & 0.4855 & 0.4827 & 0.4884 & 0.4906 & 0.4873 \\
\hline 2 & $P_{\mathrm{G} 5}$ & 0.2131 & 0.2134 & 0.2144 & 0.2137 & 0.2166 & 0.2176 & 0.2127 & 0.2158 & 0.2119 \\
\hline 3 & $P_{\mathrm{G} 8}$ & 0.2078 & 0.2059 & 0.2093 & 0.2228 & 0.2188 & 0.2253 & 0.2044 & 0.2087 & 0.2149 \\
\hline 4 & $P_{\mathrm{G} 11}$ & 0.1186 & 0.1195 & 0.1192 & 0.1253 & 0.1243 & 0.1227 & 0.1221 & 0.1143 & 0.1159 \\
\hline 5 & $P_{\mathrm{G} 13}$ & 0.12 & 0.12 & 0.12 & 0.12 & 0.12 & 0.12 & 0.12 & 0.1201 & 0.1201 \\
\hline \multicolumn{11}{|c|}{ Generator voltage } \\
\hline 6 & $V_{\mathrm{G} 1}$ & 1.1 & 1.0933 & 1.0944 & 1.0444 & 1.0504 & 1.0492 & 1.0909 & 1.0941 & 1.0948 \\
\hline 7 & $V_{\mathrm{G} 2}$ & 1.0707 & 1.0751 & 1.0752 & 1.0253 & 1.032 & 1.0319 & 1.0748 & 1.0745 & 1.0758 \\
\hline 8 & $V_{\mathrm{G} 5}$ & 1.0299 & 1.0441 & 1.0444 & 1.0078 & 1.0122 & 1.0118 & 1.0475 & 1.0435 & 1.043 \\
\hline 9 & $V_{\mathrm{G} 8}$ & 1.0405 & 1.0491 & 1.0486 & 1.0044 & 1.0059 & 1.0072 & 1.052 & 1.0489 & 1.0493 \\
\hline 10 & $V_{\mathrm{G} 11}$ & 1.1 & 1.1 & 1.0994 & 1.0751 & 1.073 & 1.0724 & 1.0999 & 1.0981 & 1.1 \\
\hline 11 & $V_{\mathrm{G} 13}$ & 1.0592 & 1.0498 & 1.0574 & 0.9904 & 0.9696 & 0.9771 & 1.0551 & 1.0558 & 1.048 \\
\hline \multicolumn{11}{|c|}{ Tap settings } \\
\hline 12 & $T_{6-9}$ & 1.1 & 1.0992 & 1.0659 & 1.1 & 1.1 & 1.1 & 1.0806 & 1.0382 & 1.1 \\
\hline 13 & $T_{6-10}$ & 0.9 & 0.9 & 0.9267 & 0.9 & 0.9 & 0.9002 & 0.9004 & 0.9451 & 0.9014 \\
\hline 14 & $T_{4-12}$ & 0.9763 & 0.9711 & 0.969 & 0.9451 & 0.9218 & 0.9229 & 0.9708 & 0.9745 & 0.9635 \\
\hline 15 & $T_{28-27}$ & 0.9813 & 0.9735 & 0.9759 & 0.9708 & 0.9699 & 0.9713 & 0.9815 & 0.9738 & 0.9821 \\
\hline \multicolumn{11}{|c|}{ Shunt VAR source } \\
\hline 16 & $Q_{\text {Sh10 }}$ & 0.0369 & 0.05 & 0.0442 & 0.05 & 0.0499 & 0.0496 & 0.0457 & 0.0214 & 0.0484 \\
\hline 17 & $Q_{\text {Sh12 }}$ & 0.0003 & 0.05 & 0.0026 & 0 & 0.05 & 0.003 & 0.0053 & 0.05 & 0.05 \\
\hline 18 & $Q_{\text {Sh15 }}$ & 0.0453 & 0.05 & 0.05 & 0.0495 & 0.05 & 0.05 & 0.0481 & 0.0335 & 0.0479 \\
\hline 19 & $Q_{\text {Sh17 }}$ & 0.05 & 0.0492 & 0.0495 & 0 & 0.0001 & 0.0003 & 0.0499 & 0.0493 & 0.0368 \\
\hline 20 & $Q_{\text {Sh20 }}$ & 0.0419 & 0.05 & 0.0414 & 0.0496 & 0.05 & 0.05 & 0.0264 & 0.046 & 0.049 \\
\hline 21 & $Q_{\text {Sh21 }}$ & 0.05 & 0.0499 & 0.05 & 0.0499 & 0.05 & 0.0498 & 0.0499 & 0.0466 & 0.0498 \\
\hline 22 & $Q_{\text {Sh23 }}$ & 0.0332 & 0.037 & 0.0352 & 0.0496 & 0.05 & 0.0497 & 0.0432 & 0.0404 & 0.0418 \\
\hline
\end{tabular}


Table 3. Cont.

\begin{tabular}{|c|c|c|c|c|c|c|c|c|c|c|}
\hline \multirow{2}{*}{$\begin{array}{c}\text { S. } \\
\text { Number }\end{array}$} & \multirow{2}{*}{$\begin{array}{c}\text { Control } \\
\text { Variable }\end{array}$} & \multicolumn{3}{|c|}{$\begin{array}{l}\text { Case } 1 \\
\text { (FCM) }\end{array}$} & \multicolumn{3}{|c|}{$\begin{array}{c}\text { Case } 2 \\
\text { (VPI) }\end{array}$} & \multicolumn{3}{|c|}{$\begin{array}{l}\text { Case } 3 \\
\text { (VSE) }\end{array}$} \\
\hline & & Rao-1 & Rao-2 & Rao-3 & Rao-1 & Rao-2 & Rao-3 & Rao-1 & Rao-2 & Rao-3 \\
\hline 23 & $Q_{\text {Sh24 }}$ & 0.05 & 0.0493 & 0.0497 & 0.05 & 0.05 & 0.0491 & 0.05 & 0.0489 & 0.0476 \\
\hline 24 & $Q_{\text {Sh29 }}$ & 0.0278 & 0.0178 & 0.029 & 0.033 & 0.0265 & 0.0285 & 0.0333 & 0.0309 & 0.05 \\
\hline \multicolumn{2}{|c|}{ Fuel cost $(\$ \backslash h)$} & 800.4391 & 799.9918 & 799.9683 & 803.4877 & 803.5375 & 803.5304 & 800.0492 & 800.001 & 800.025 \\
\hline \multicolumn{2}{|c|}{ TVD (pu) } & 0.9714 & 1.1168 & 1.1356 & 0.1031 & 0.0993 & 0.1001 & 1.1481 & 1.1409 & 1.1449 \\
\hline \multicolumn{2}{|c|}{ Emission (ton/h) } & 0.3362 & 0.3351 & 0.3351 & 0.3315 & 0.3338 & 0.3331 & 0.3357 & 0.3355 & 0.3354 \\
\hline \multicolumn{2}{|c|}{$P_{\text {Loss }}(\mathrm{MW})$} & 9.0613 & 8.91 & 8.8872 & 9.7465 & 9.8209 & 9.7724 & 8.941 & 8.9086 & 8.9098 \\
\hline \multicolumn{2}{|c|}{ L-index (LI) } & 0.1307 & 0.1285 & 0.1281 & 0.1404 & 0.1404 & 0.1408 & 0.128 & 0.1278 & 0.1264 \\
\hline
\end{tabular}

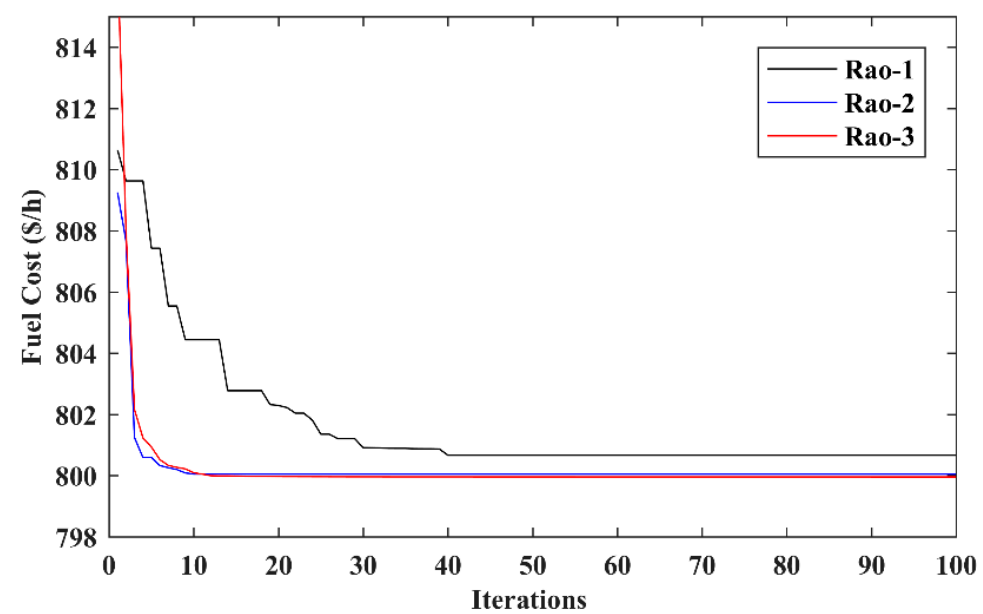

Figure 2. Convergence characteristics for the IEEE 30-bus system, Case 1.

\subsubsection{Case 2: Voltage Profile Improvement (VPI)}

The main motive of the second case is to minimize the voltage variation in all the load buses from $1.0 \mathrm{pu}$ [31] along with the fuel cost. The multi-objective function is transformed into a single objective function by using the weighted sum method using Equation (22):

$$
F_{T V D}(a, b)=\sum_{i=1}^{N G N} f_{i}(\cdot) \$ / h+\varphi_{V D} \sum_{i \in N L B}\left|V_{i}-1\right|
$$

In the combined objective function, the value of the weighting factor $\left(\varphi_{V D}\right)$ assigned to voltage deviation was taken as 160 .

The OPF results attained using Rao algorithms are compared with other reported results in Table 4. As can be observed from Table 4, the Rao-2 algorithm provided the minimum total voltage deviation as $0.0993 \mathrm{pu}$, which is the least among the Rao algorithm variants. In this case, the minimum total voltage deviation obtained by the proposed Rao algorithms are slightly higher than some reported results. Such types of situations will be there only because the different papers might have selected different weighting factors.

The optimal control variable settings are presented in Table 3, while the load (PQ) bus voltage profile obtained by the Rao-2 algorithm in Case 2 is shown in Figure 3. Based on the OPF results, it is clear that the Rao-2 algorithm provided the least value of the total voltage deviation as compared to the other variants of the Rao method. This demonstrates the effectiveness of the Rao- 2 algorithm in comparison to the Rao- 1 and Rao- 3 algorithms for this case. 
Table 4. Case 2: VPI in the IEEE 30-bus system.

\begin{tabular}{cccc}
\hline Algorithm & Fuel Cost (\$/h) & TVD (pu) & Time (s) \\
\hline Rao-3 & 803.5304 & 0.1001 & 88.14 \\
\hline Rao-2 & 803.5375 & 0.0993 & 87.36 \\
\hline Rao-1 & 803.4877 & 0.1031 & - \\
\hline MSA [14] & 803.3125 & 0.1084 & - \\
\hline MPSO [14] & 803.9787 & 0.1202 & - \\
\hline MDE [14] & 803.2122 & 0.1265 & - \\
\hline MFO [14] & 803.7911 & 0.1056 & - \\
\hline FPA [14] & 803.6638 & 0.1366 & - \\
\hline IMFO [38] & 803.5715 & 0.0954 & - \\
\hline MFO [38] & 803.5173 & 0.1007 & - \\
\hline GA [38] & 803.2347 & 0.1018 & 123.3 \\
\hline PSO [38] & 803.4736 & 0.0978 & - \\
\hline TLBO [38] & 803.5675 & 0.0939 & - \\
\hline ECHT-DE [44] & 803.7198 & 0.09454 & - \\
\hline SF-DE [44] & 803.4241 & 0.09772 & - \\
\hline SP-DE [44] & 803.4196 & 0.09776 & 0.1107 \\
\hline MGOA [45] & 803.4176 & 0.1709 & - \\
\hline GOA [45] & 803.4488 & & - \\
\hline
\end{tabular}

TVD = total voltage deviation.

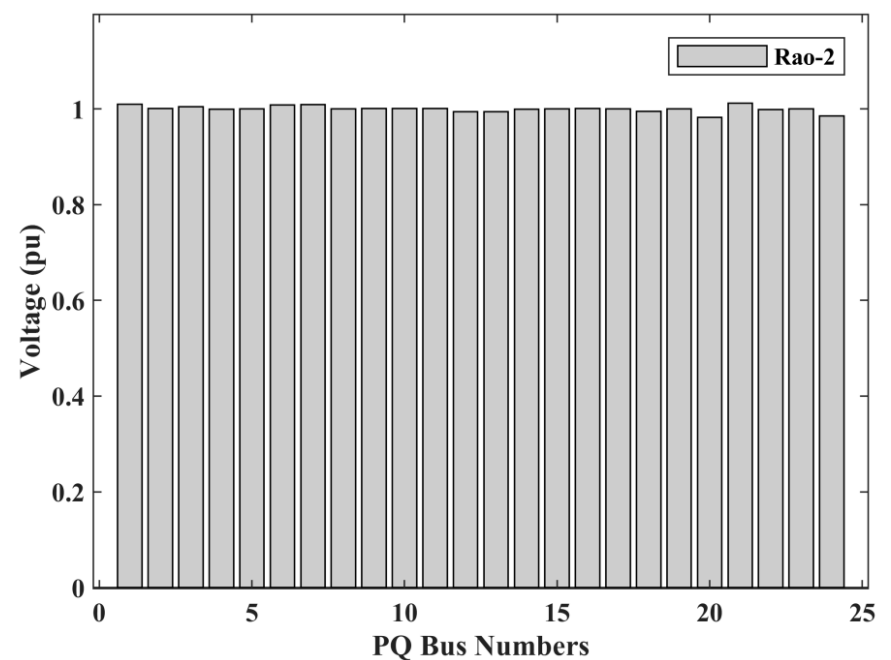

Figure 3. Load bus voltage profile for the IEEE 30-bus system, Case 2.

\subsubsection{Case 3: Voltage Stability Enhancement (VSE)}

The system voltage stability can be increased by reducing the L-index [8]. In this paper, fuel cost minimization and voltage stability enhancement were considered using the two-fold objective function as in Equation (23):

$$
F_{L \max }(a, b)=\sum_{i=1}^{N G N} f_{i}(\cdot) \$ / h+\varphi_{L} L
$$

where $\varphi_{L}$ is the weight factor.

The control variables' settings of this case obtained using the three variants of Rao algorithms are given in Table 5. The OPF results of Rao algorithms and the results provided 
by other reported results published in recent literature have been compared in Table 5 . The OPF results in Table 5 prove that the Rao- 3 algorithm is best among three variants of Rao algorithms for Case 3. As can be observed from Table 5, the Rao-3 algorithm provided a minimum L-index value of 0.1264 , which is the least among the Rao variants. In this case, the minimum L-index values obtained by the proposed Rao algorithms are slightly higher than some reported results but the fuel cost is less. Such types of situations will be there only because the different papers might have selected different weighting factors.

Table 5. Case 3: VSE in the IEEE 30-bus system.

\begin{tabular}{cccc}
\hline Algorithm & Fuel Cost (\$/h) & L-Index & Time (s) \\
\hline Rao-3 & 800.0250 & 0.1264 & 87.94 \\
\hline Rao-2 & 800.0010 & 0.1278 & 88.50 \\
\hline Rao-1 & 800.0492 & 0.1280 & 88.11 \\
\hline MSA [14] & 801.2248 & 0.1371 & - \\
\hline MPSO [14] & 801.6966 & 0.1375 & - \\
\hline MDE [14] & 802.0991 & 0.1374 & - \\
\hline MFO [14] & 801.668 & 0.1376 & - \\
\hline FPA [14] & 801.1487 & 0.1376 & - \\
\hline IMFO [38] & 800.4762 & 0.1255 & - \\
\hline MFO [38] & 800.9415 & 0.1266 & - \\
\hline GA [38] & 800.4385 & 0.1254 & 130.4 \\
\hline PSO [38] & 800.5815 & 0.128 & - \\
\hline TLBO [38] & 800.4738 & 0.1247 & - \\
\hline ECHT-DE [44] & 800.4321 & 0.13739 & - \\
\hline SF-DE [44] & 800.4203 & 0.13745 & - \\
\hline SP-DE [44] & 800.4365 & 0.13748 & -1050 \\
\hline Bisection method [47] & 958.8330 & & - \\
\hline -index = voltage collapse proximity indicator. & & \\
\hline
\end{tabular}

\subsubsection{Case 4: Voltage Stability Enhancement (VSE) during Contingency}

In Case 4 , voltage stability has been improved considering single line outage $(n-1)$ contingency [8]. The prime objective in this case is to improve voltage stability and reduce the fuel cost under a single line (connected between bus number 2 to bus number 6 ) outage condition. Table 6 compares the OPF results of Case 4 obtained by the proposed Rao algorithms with other efficient optimization algorithms reported in the recent literature. The results shown in Table 6 demonstrate the Rao- 3 algorithm's dominance over other recently developed optimization methods. The control variables' settings obtained in this case using the three Rao algorithms are given in Table 7.

Table 6. Case 4: VSE during contingency results in the IEEE 30-bus system.

\begin{tabular}{cccc}
\hline Algorithm & Fuel Cost (\$/h) & L-Index & Time (s) \\
\hline Rao-3 & 818.5353 & 0.1363 & 90.65 \\
\hline Rao-2 & 810.3012 & 0.1439 & 92.32 \\
\hline Rao-1 & 827.3375 & 0.1485 & 91.87 \\
\hline MSA [14] & 804.4838 & 0.1392 & - \\
\hline MPSO [14] & 807.6519 & 0.1405 & - \\
\hline MDE [14] & 806.6668 & 0.1398 & - \\
\hline
\end{tabular}


Table 6. Cont.

\begin{tabular}{cccc}
\hline Algorithm & Fuel Cost (\$/h) & L-Index & Time (s) \\
\hline MFO [14] & 804.556 & 0.1394 & - \\
\hline FPA [14] & 805.5446 & 0.1415 & - \\
\hline
\end{tabular}

Table 7. Optimum values of control variables of Case 4, Case 5, and Case 6 of the IEEE 30-bus system.

\begin{tabular}{|c|c|c|c|c|c|c|c|c|c|c|}
\hline \multirow{2}{*}{$\begin{array}{c}\text { S. } \\
\text { Number }\end{array}$} & \multirow{2}{*}{$\begin{array}{c}\text { Control } \\
\text { Variable(p.u) }\end{array}$} & \multicolumn{3}{|c|}{$\begin{array}{c}\text { Case } 4 \\
\text { (VSE) during Contingency }\end{array}$} & \multicolumn{3}{|c|}{$\begin{array}{l}\text { Case } 5 \\
\text { (RPLM) }\end{array}$} & \multicolumn{3}{|c|}{$\begin{array}{l}\text { Case } 6 \\
\text { (ECM) }\end{array}$} \\
\hline & & Rao-1 & Rao-2 & Rao-3 & Rao-1 & Rao-2 & Rao-3 & Rao-1 & Rao-2 & Rao-3 \\
\hline \multicolumn{11}{|c|}{ Generator active power output } \\
\hline 1 & $P_{\mathrm{G} 2}$ & 0.4034 & 0.4515 & 0.4811 & 0.8 & 0.8 & 0.8 & 0.6633 & 0.6631 & 0.7421 \\
\hline 2 & $P_{\mathrm{G} 5}$ & 0.2951 & 0.2132 & 0.214 & 0.5 & 0.5 & 0.5 & 0.5 & 0.5 & 0.4638 \\
\hline 3 & $P_{\mathrm{G} 8}$ & 0.2935 & 0.2808 & 0.2416 & 0.35 & 0.35 & 0.35 & 0.35 & 0.35 & 0.3154 \\
\hline 4 & $P_{\mathrm{G} 11}$ & 0.168 & 0.12 & 0.1279 & 0.3 & 0.3 & 0.3 & 0.3 & 0.3 & 0.2867 \\
\hline 5 & $P_{\mathrm{G} 13}$ & 0.2854 & 0.12 & 0.12 & 0.4 & 0.4 & 0.4 & 0.4 & 0.4 & 0.3127 \\
\hline \multicolumn{11}{|c|}{ Generator voltage } \\
\hline 6 & $V_{\mathrm{G} 1}$ & 1.0414 & 1.1 & 1.02 & 1.066 & 1.0616 & 1.0718 & 1.0737 & 0.9963 & 1.0473 \\
\hline 7 & $V_{\mathrm{G} 2}$ & 1.0035 & 1.469 & 1.02 & 1.0509 & 1.0577 & 1.0679 & 1.0677 & 0.95 & 1.0441 \\
\hline 8 & $V_{\mathrm{G} 5}$ & 1.0416 & 1.095 & 1.092 & 1.025 & 1.0381 & 1.0484 & 1.0477 & 0.956 & 1.0317 \\
\hline 9 & $V_{\mathrm{G} 8}$ & 1.0799 & 1.095 & 1.08 & 1.0409 & 1.0495 & 1.0552 & 1.0539 & 1.0932 & 1.0488 \\
\hline 10 & $V_{\mathrm{G} 11}$ & 1.0602 & 1.06 & 1.1 & 1.02 & 1.1 & 1.1 & 1.1 & 0.9539 & 1.0924 \\
\hline 11 & $V_{\mathrm{G} 13}$ & 1.0237 & 1.0714 & 1.1 & 1.0452 & 1.07 & 1.063 & 1.0613 & 1.1 & 1.0719 \\
\hline \multicolumn{11}{|c|}{ Tap settings } \\
\hline 12 & $T_{6-9}$ & 0.9602 & 1.0496 & 0.914 & 1.1 & 1.0858 & 1.0822 & 1.0445 & 0.9063 & 1.0493 \\
\hline 13 & $T_{6-10}$ & 1.0208 & 1.0657 & 0.9747 & 0.9108 & 0.9001 & 0.9 & 0.9518 & 0.9054 & 1.0764 \\
\hline 14 & $T_{4-12}$ & 1.0379 & 1.0012 & 0.9549 & 1.0307 & 0.9977 & 0.9966 & 0.9928 & 0.9762 & 1.0378 \\
\hline 15 & $T_{28-27}$ & 0.9724 & 0.9327 & 0.9256 & 1.0072 & 0.9772 & 0.9774 & 0.9761 & 0.9156 & 1.0724 \\
\hline \multicolumn{11}{|c|}{ Shunt VAR source } \\
\hline 16 & $Q_{\text {Sh10 }}$ & 0.021 & 0.05 & 0.05 & 0.05 & 0 & 0 & 0.0293 & 0.0317 & 0.0217 \\
\hline 17 & $Q_{\text {Sh12 }}$ & 0.0324 & 0.05 & 0.05 & 0.045 & 0.0479 & 0.0478 & 0.05 & 0.0414 & 0.0429 \\
\hline 18 & $Q_{\text {Sh15 }}$ & 0.032 & 0.05 & 0.05 & 0.0495 & 0.039 & 0.0471 & 0.0448 & 0 & 0.0342 \\
\hline 19 & $Q_{\text {Sh17 }}$ & 0.0151 & 0.05 & 0.05 & 0.05 & 0.0499 & 0.0498 & 0.05 & 0.0349 & 0.0343 \\
\hline 20 & $Q_{\text {Sh20 }}$ & 0.0283 & 0.05 & 0.05 & 0.05 & 0.0413 & 0.0412 & 0.0413 & 0.0018 & 0.0122 \\
\hline 21 & $Q_{\text {Sh21 }}$ & 0.0344 & 0.05 & 0.05 & 0.05 & 0.05 & 0.05 & 0.05 & 0.05 & 0.0433 \\
\hline 22 & $Q_{\text {Sh23 }}$ & 0.0147 & 0.05 & 0.05 & 0.0427 & 0.0371 & 0.0341 & 0.0332 & 0.05 & 0.0358 \\
\hline 23 & $Q_{\text {Sh24 }}$ & 0.0282 & 0.05 & 0.05 & 0.05 & 0.05 & 0.05 & 0.05 & 0.05 & 0.0458 \\
\hline 24 & $Q_{\text {Sh29 }}$ & 0.0232 & 0.05 & 0.05 & 0.0205 & 0.0257 & 0.0252 & 0.0236 & 0.05 & 0.0383 \\
\hline \multicolumn{2}{|c|}{ Fuel cost $(\$ \backslash h)$} & 827.3375 & 810.3012 & 818.5353 & 968.1496 & 967.6830 & 967.5828 & 942.3443 & 944.1722 & 915.2185 \\
\hline \multicolumn{2}{|c|}{ TVD (pu) } & 0.5925 & 0.5754 & 0.7439 & 0.4125 & 1.0361 & 1.1277 & 1.1261 & 0.821 & 0.5493 \\
\hline \multicolumn{2}{|c|}{ Emission (ton/h) } & 0.2792 & 0.3324 & 0.3375 & 0.2066 & 0.2066 & 0.2066 & 0.2037 & 0.204 & 0.2126 \\
\hline \multicolumn{2}{|c|}{$P_{\text {Loss }}(\mathrm{MW})$} & 9.2645 & 11.3868 & 14.145 & 3.3041 & 3.1086 & 3.0675 & 3.2162 & 3.9623 & 4.2325 \\
\hline \multicolumn{2}{|c|}{ L-index } & 0.1485 & 0.1439 & 0.1363 & 0.1391 & 0.1302 & 0.1289 & 0.1286 & 0.1328 & 0.1467 \\
\hline
\end{tabular}




\subsubsection{Case 5: Real Power Loss Minimization (RPLM)}

In Case 5, RPLM was selected as a prime objective function [29]. Mathematically, $F_{\text {Loss }}$ can be represented by Equation (24).

$$
F_{\text {Loss }}(a, b)=\sum_{i=1}^{N B} P_{g i}-\sum_{i=1}^{N B} P_{d i}
$$

The function $F_{\text {Loss }}$ is selected for the power loss as described in Equation (24). The minimum power loss attained by the Rao-3 algorithm is 3.0675 MW, while Rao-2 and Rao-1 algorithms provided minimum power losses of $3.1086 \mathrm{MW}$ and 3.3041 MW, respectively. The results of Rao algorithms and optimal control variable settings are presented in Table 7. Table 8 compares the simulation results of Case 5 obtained by the proposed algorithms and other published methods proposed in recent literature. Based on the OPF outcomes, it can be concluded that the Rao-3 algorithm provided the least value of power loss as compared to the other methods. The power loss convergence characteristics of Case 5 are presented in Figure 4.

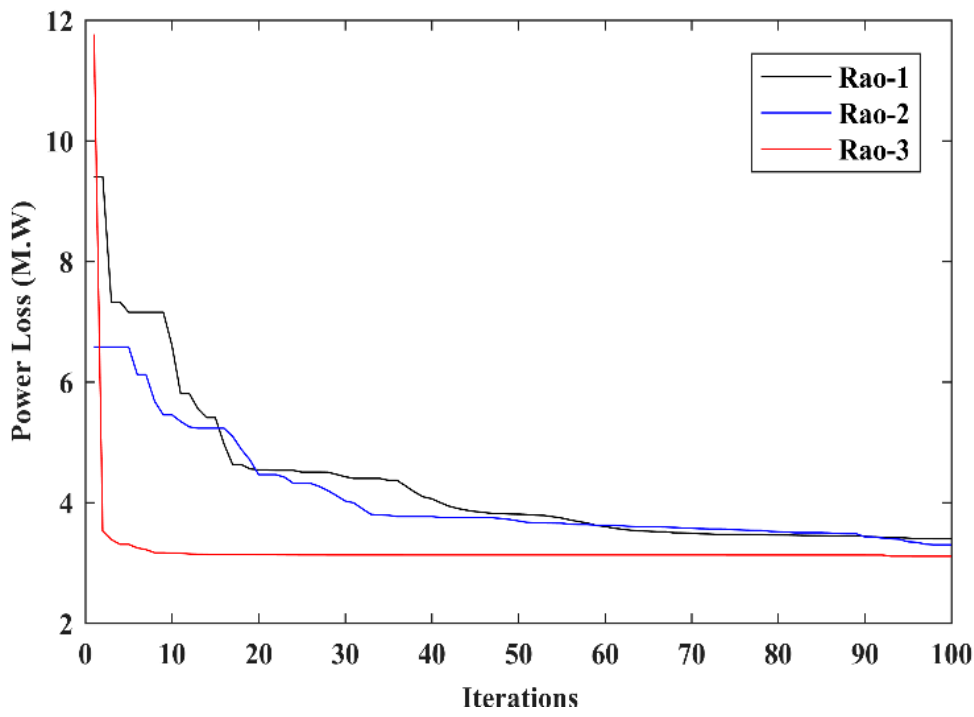

Figure 4. Convergence characteristics for the IEEE 30-bus system, Case 5.

Table 8. Case 5: RPLM in the IEEE 30-bus system.

\begin{tabular}{ccc}
\hline Algorithm & Power Loss (MW) & Time (s) \\
\hline Rao-3 & 3.0675 & 85.72 \\
\hline Rao-2 & 3.1086 & 90.89 \\
\hline Rao-1 & 3.3041 & 89.07 \\
\hline MSA [14] & 3.1005 & - \\
\hline MPSO [14] & 3.1031 & - \\
\hline MDE [14] & 3.1619 & - \\
\hline MFO [14] & 3.1111 & - \\
\hline FPA [14] & 3.5661 & - \\
\hline MSO [37] & 3.4052 & - \\
\hline IMFO [38] & 3.0905 & - \\
\hline MFO [38] & 3.139 & - \\
\hline GA [38] & 3.118 &
\end{tabular}


Table 8. Cont.

\begin{tabular}{ccc}
\hline Algorithm & Power Loss (MW) & Time (s) \\
\hline PSO [38] & 3.103 & - \\
\hline TLBO [38] & 3.088 & - \\
\hline SKH [43] & 3.0987 & - \\
\hline ECHT-DE [44] & 3.0850 & - \\
\hline SF-DE [44] & 3.0845 & - \\
\hline SP-DE [44] & 3.0844 & 136.4 \\
\hline
\end{tabular}

\subsubsection{Case 6: Emission Cost Minimization}

For a given electrical network, the total emission cost [29] can be calculated using Equation (25):

$$
F_{E C O S T}(x, u)=\sum_{i=1}^{N G N} \delta_{i}+\mu_{i} P_{G_{i}}+\gamma_{i} P_{G_{i}}^{2}+\xi_{i} \exp \left(\lambda_{i} P_{G_{i}}\right)\left(\frac{\text { ton }}{h}\right)
$$

The objective function $F_{E C O S T}$ is selected for total emission cost minimization as described in Equation (25). Table 9 compares the results obtained by the proposed Rao algorithms for Case 6 with other efficient optimization algorithms reported in the recent literature. The results shown in Table 9 demonstrate the dominance of the Rao- 1 algorithm over other variants of Rao techniques. The convergence characteristics offered by the three Rao algorithms are shown in Figure 5. The control variables' settings for Case 6 obtained using the three Rao algorithms are given in Table 7. As can be noted from Table 9, the MGOA [45] algorithm offered the least emission value in comparison to the Rao-1 algorithm in this case, but Rao algorithm variants offered better results in most of the cases.

Table 9. Case 6: ECM in the IEEE 30-bus system.

\begin{tabular}{ccc}
\hline Algorithm & Emission (ton/h) & Time (s) \\
\hline Rao-3 & 0.2126 & 87.87 \\
\hline Rao-2 & 0.2040 & 85.45 \\
\hline Rao-1 & 0.2037 & 89.82 \\
\hline MSA [14] & 0.2048 & - \\
\hline MPSO [14] & 0.2325 & - \\
\hline MDE [14] & 0.2093 & - \\
\hline MFO [14] & 0.2049 & - \\
\hline FPA [14] & 0.2052 & - \\
\hline DSA [18] & 0.2058 & - \\
\hline MSO [37] & 0.2175 & - \\
\hline IMFO [38] & 0.2048 & - \\
\hline MFO [38] & 0.2048 & - \\
\hline GA [38] & 0.2048 & - \\
\hline PSO [38] & 0.2048 & - \\
\hline TLBO [38] & 0.2048 & - \\
\hline MGBICA [41] & 0.2048 & - \\
\hline GBICA [41] & 0.2049 & - \\
\hline
\end{tabular}


Table 9. Cont.

\begin{tabular}{ccc}
\hline Algorithm & Emission (ton/h) & Time (s) \\
\hline ABC [42] & 0.2048 & - \\
\hline SKH [43] & 0.2048 & - \\
\hline ECHT-DE [44] & 0.2048 & 138.2 \\
\hline SF-DE [44] & 0.2048 & - \\
\hline SP-DE [44] & 0.2048 & - \\
\hline MGOA [45] & 0.2025 & - \\
\hline GOA [45] & 0.2050 & - \\
\hline
\end{tabular}

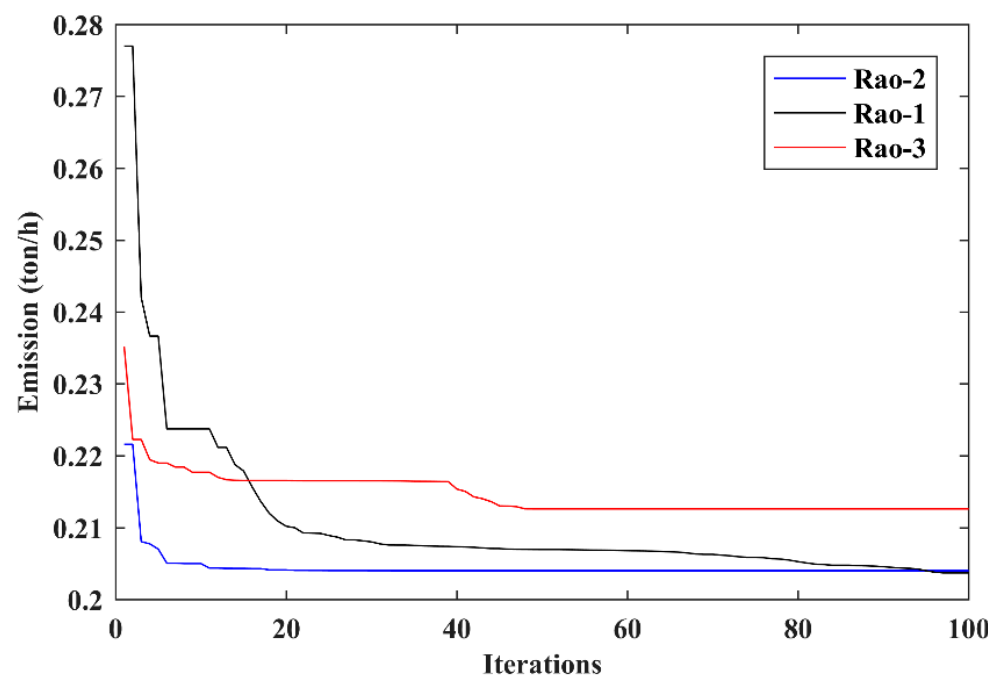

Figure 5. Convergence characteristic for the IEEE 30-bus system, Case 6.

\subsection{IEEE 57-Bus System}

To evaluate the effectiveness of the Rao algorithms, they are applied to the IEEE 57-bus system to solve OPF problems. The system data, shunt capacitor data, transformer data, and control variables' limits are taken from [48] and are shown in Table 10. The active and reactive power demands of this system on the 100 MVA base are 12.508 and $3.364 \mathrm{pu}$, respectively. Thirty independent runs were taken using Rao algorithms to solve the OPF problem for this system and the best results obtained are given in this paper.

Table 10. Details of the IEEE 57-bus system.

\begin{tabular}{|c|c|c|c|c|c|c|c|c|}
\hline $\begin{array}{c}\text { Control } \\
\text { Variable }\end{array}$ & Nbus & $N t l$ & $N G N$ & $N L B$ & $N C$ & NTR & $\begin{array}{c}\text { Voltage } \\
\text { Limit } \\
\text { (PQ Bus) }\end{array}$ & $\begin{array}{c}\text { Voltage } \\
\text { Limit } \\
\text { (PV Bus) }\end{array}$ \\
\hline $\begin{array}{c}33 \\
(06+07+03+17)\end{array}$ & 57 & 80 & $\begin{array}{c}7\left(@ \mathrm{G}_{1}, \mathrm{G}_{2}\right. \\
\mathrm{G}_{3} \mathrm{G}_{6}, \mathrm{G}_{8} \mathrm{G}_{9} \\
\left.\mathrm{G}_{12}\right)\end{array}$ & 50 & $\begin{array}{c}\text { 3(@ Sh18, } \\
\text { Sh25, Sh23) }\end{array}$ & $\begin{array}{c}17 \text { (@ Nt19, Nt20, Nt31 Nt } \\
\text { 35, Nt 36, Nt 37, Nt 41, } \\
\text { Nt46, Nt54, Nt58, Nt59, } \\
\text { Nt65, Nt66, Nt71, Nt73, } \\
\text { Nt76 \& Nt80) }\end{array}$ & $(0.94: 1.06)$ & $(0.9: 1.1)$ \\
\hline
\end{tabular}

\subsubsection{Case 7: Fuel Cost Minimization (FCM)}

FCM is selected as the primary objective as in Case 1 and defined in Equation (20). Table 11 compares the simulation results of Case 7 as obtained by the proposed Rao algorithms and by other methods reported in recent literature. The minimum cost attained by the Rao-3 algorithm is $41,659.2621 \$ / \mathrm{h}$, while Rao-2 and Rao-1 algorithms offered 
the minimum fuel cost of $41,872.0668 \$ / \mathrm{h}$ and $41,771.1088 \$ / \mathrm{h}$, respectively. Based on the OPF outcomes, it is clear that the Rao-3 algorithm provided the least fuel cost as compared to other methods. This demonstrates the effectiveness of the proposed Rao-3 algorithm as compared to Rao-2 and Rao-1 algorithms, as well as other reported algorithms. The OPF results of the proposed Rao- 3 algorithm and optimal control variable settings are presented in Table 12. The fuel cost characteristic of Case 7 is presented in Figure 6.

Table 11. Case 7 (FCM) results in the IEEE 57-bus system.

\begin{tabular}{|c|c|c|}
\hline Algorithm & Fuel Cost $(\$ / h)$ & Time (s) \\
\hline Rao-3 & $41,659.2621$ & 131.23 \\
\hline Rao-2 & $41,872.0668$ & 132.94 \\
\hline Rao-1 & $41,771.1088$ & 131.87 \\
\hline MSA [14] & $41,673.7231$ & - \\
\hline MPSO [14] & $41,678.6762$ & - \\
\hline MDE [14] & $41,695.8123$ & - \\
\hline MFO [14] & $41,686.4119$ & - \\
\hline FPA [14] & $41,701.9592$ & - \\
\hline TSA [17] & $41,685.07$ & 75.61 \\
\hline DSA [18] & $41,686.82$ & - \\
\hline SSA [19] & $41,672.30$ & 80.61 \\
\hline MSO [37] & $41,747.20$ & - \\
\hline IMFO [38] & $41,692.7178$ & - \\
\hline MFO [38] & $41,719.8471$ & - \\
\hline GA [38] & $41,700.4162$ & - \\
\hline PSO [38] & $41,684.4009$ & - \\
\hline TLBO [38] & $41,694.7778$ & - \\
\hline SKH [43] & $41,676.9152$ & - \\
\hline ECHT-DE [44] & $41,670.562$ & - \\
\hline SF-DE [44] & $41,667.85$ & - \\
\hline SP-DE [44] & $41,667.82$ & 219.9 \\
\hline MGOA [45] & $41,671.0980$ & - \\
\hline GOA [45] & $41,679.6792$ & - \\
\hline
\end{tabular}

Table 12. Optimum values of control variables of Case 7 and Case 8 for the IEEE 57-bus system.

\begin{tabular}{|c|c|c|c|c|c|c|c|}
\hline \multirow[t]{2}{*}{ S. Number } & \multirow{2}{*}{$\begin{array}{c}\text { Control Variable } \\
\text { (p.u) }\end{array}$} & \multicolumn{3}{|c|}{$\begin{array}{l}\text { CASE } 7 \\
\text { (FCM) }\end{array}$} & \multicolumn{3}{|c|}{$\begin{array}{c}\text { CASE } 8 \\
\text { (VPI) }\end{array}$} \\
\hline & & Rao-1 & Rao-2 & Rao-3 & Rao-1 & Rao-2 & Rao-3 \\
\hline \multicolumn{8}{|c|}{ Generator active power output } \\
\hline 1 & $P_{\mathrm{G} 2}$ & 0.8722 & 0.9999 & 0.8857 & 0.8822 & 0.8866 & 0.4027 \\
\hline 2 & $P_{\mathrm{G} 3}$ & 0.42 & 0.5217 & 0.4494 & 0.4506 & 0.4497 & 0.42 \\
\hline 3 & $P_{\mathrm{G} 6}$ & 0.7856 & 0.3264 & 0.7324 & 0.7298 & 0.7183 & 0.3135 \\
\hline 4 & $P_{\mathrm{G} 8}$ & 4.6615 & 4.5567 & 4.6028 & 4.6168 & 4.5992 & 4.814 \\
\hline 5 & $P_{\mathrm{G} 9}$ & 0.8309 & 0.94 & 0.9588 & 0.963 & 0.9726 & 0.962 \\
\hline 6 & $P_{\mathrm{G} 12}$ & 3.639 & 3.9341 & 3.5953 & 3.5936 & 3.607 & 4.087 \\
\hline
\end{tabular}


Table 12. Cont.

\begin{tabular}{|c|c|c|c|c|c|c|c|}
\hline \multirow[t]{2}{*}{ S. Number } & \multirow{2}{*}{$\begin{array}{c}\text { Control Variable } \\
\text { (p.u) }\end{array}$} & \multicolumn{3}{|c|}{$\begin{array}{l}\text { CASE } 7 \\
\text { (FCM) }\end{array}$} & \multicolumn{3}{|c|}{$\begin{array}{c}\text { CASE } 8 \\
\text { (VPI) }\end{array}$} \\
\hline & & Rao-1 & Rao-2 & Rao-3 & Rao-1 & Rao-2 & Rao-3 \\
\hline \multicolumn{8}{|c|}{ Generator voltage } \\
\hline 7 & $V_{\mathrm{G} 1}$ & 1.0791 & 1.0629 & 1.0603 & 1.0484 & 1.0322 & 0.9965 \\
\hline 8 & $V_{\mathrm{G} 2}$ & 1.0822 & 1.0694 & 1.0637 & 1.0526 & 1.0362 & 1.014 \\
\hline 9 & $V_{\mathrm{G} 3}$ & 1.0602 & 1.0556 & 1.0529 & 1.043 & 1.0255 & 1.0097 \\
\hline 10 & $V_{\mathrm{G} 6}$ & 1.0611 & 1.0493 & 1.0615 & 1.057 & 1.04 & 1.0032 \\
\hline 11 & $V_{\mathrm{G} 8}$ & 1.0656 & 1.0626 & 1.0741 & 1.0757 & 1.0592 & 1.0135 \\
\hline 12 & $V_{\mathrm{G} 9}$ & 1.0508 & 1.0484 & 1.0541 & 1.0502 & 1.0329 & 1.0148 \\
\hline 13 & $V_{\mathrm{G} 12}$ & 1.0518 & 1.046 & 1.0462 & 1.0342 & 1.0175 & 1.044 \\
\hline \multicolumn{8}{|c|}{ Tap settings } \\
\hline 14 & $T_{4-18}$ & 1.0824 & 1.001 & 1.1 & 0.982 & 1.0872 & 0.9031 \\
\hline 15 & $T_{4-18}$ & 1.0075 & 1.0173 & 0.9416 & 1.0113 & 0.9243 & 1.0393 \\
\hline 16 & $T_{21-20}$ & 1.0187 & 1.0649 & 1.0154 & 0.9892 & 0.991 & 0.9757 \\
\hline 17 & $T_{24-25}$ & 1.0879 & 1.0289 & 0.9447 & 1.017 & 0.9452 & 1.1 \\
\hline 18 & $T_{24-25}$ & 1.0887 & 0.9164 & 1.0887 & 1.0503 & 1.0952 & 1.0996 \\
\hline 19 & $T_{24-26}$ & 1.0277 & 0.9031 & 1.0327 & 1.1 & 1.0224 & 1.0152 \\
\hline 20 & $T_{7-29}$ & 1.0149 & 1.0082 & 0.9954 & 1.034 & 1.014 & 1.0054 \\
\hline 21 & $T_{34-32}$ & 1.0011 & 0.9549 & 0.9565 & 0.938 & 0.9356 & 0.9334 \\
\hline 22 & $T_{11-41}$ & 1.0006 & 0.9111 & 0.9083 & 0.9 & 0.9008 & 0.9002 \\
\hline 23 & $T_{15-45}$ & 1.01 & 1.1 & 0.9781 & 0.989 & 0.9691 & 0.9524 \\
\hline 24 & $T_{14-46}$ & 0.9841 & 0.9489 & 0.9612 & 0.9866 & 0.9651 & 0.9798 \\
\hline 25 & $T_{10-51}$ & 1.0997 & 0.9788 & 0.9748 & 1.0039 & 0.9848 & 1.0138 \\
\hline 26 & $T_{13-49}$ & 0.9037 & 0.9328 & 0.936 & 0.9553 & 0.9357 & 0.9001 \\
\hline 27 & $T_{11-43}$ & 1.0938 & 1.0018 & 0.9771 & 1.0047 & 0.9745 & 0.9781 \\
\hline 28 & $T_{40-56}$ & 0.9067 & 0.9 & 0.9975 & 1.0041 & 0.9975 & 0.9849 \\
\hline 29 & $T_{39-57}$ & 0.9182 & 0.9 & 0.9675 & 0.9415 & 0.9384 & 0.9 \\
\hline 30 & $T_{9-55}$ & 1.0134 & 1.1 & 1.0026 & 1.0285 & 1.0115 & 1.0146 \\
\hline \multicolumn{8}{|c|}{ Shunt VAR source } \\
\hline 31 & $\mathrm{Q}_{\mathrm{sh} 18}$ & 0.1858 & 0.0559 & 0.1724 & 0.0127 & 0.0628 & 0.0003 \\
\hline 32 & $\mathrm{Q}_{\text {sh25 }}$ & 0.2803 & 0.1939 & 0.1439 & 0.163 & 0.1747 & 0.3 \\
\hline 33 & $\mathrm{Q}_{\text {sh53 }}$ & 0.2381 & 0.1577 & 0.1267 & 0.1705 & 0.1481 & 0.3 \\
\hline \multicolumn{2}{|c|}{ Fuel cost $(\$ \backslash h)$} & $41,771.1088$ & $41,872.0668$ & $41,659.2621$ & $41,688.4417$ & $41,691.1102$ & $42,043.2728$ \\
\hline \multicolumn{2}{|c|}{ TVD (pu) } & 1.5465 & 1.6713 & 1.6953 & 0.9882 & 0.7645 & 0.5725 \\
\hline \multicolumn{2}{|c|}{ L-index } & 0.231 & 0.2411 & 0.2349 & 0.2438 & 0.2415 & 0.2297 \\
\hline \multicolumn{2}{|c|}{$P_{\text {Loss }}(\mathrm{MW})$} & 17.364 & 16.4837 & 14.7262 & 15.4719 & 15.4214 & 18.0100 \\
\hline
\end{tabular}




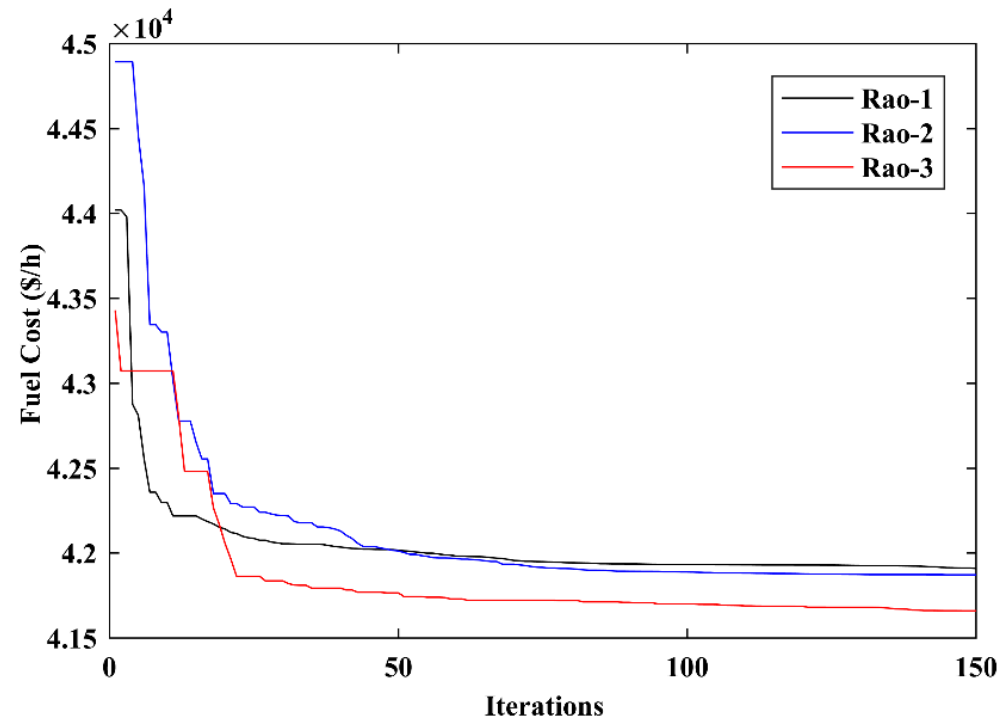

Figure 6. Convergence characteristics for the IEEE 57-bus system, Case 7.

\subsubsection{Case 8: Voltage Profile Improvement (VPI)}

In Case 8 , improvement of the voltage profile is considered as an objective function in addition to the fuel cost, as defined in Equation (22). The optimal control variables' settings are presented in Table 12. The minimum total voltage deviation attained by the Rao-3 algorithm is $0.5725 \mathrm{pu}$, while Rao- 1 and Rao-2 algorithms provided the minimum total voltage deviations of 0.9882 and $0.9882 \mathrm{pu}$, respectively. Table 13 compares the simulation results of Case 8 obtained by the proposed Rao algorithms and other proposed methods reported in recent literature. Based on the OPF outcomes shown in Table 13, it can be concluded that the Rao-3 algorithm provided the least total voltage deviation compared to the other methods. This demonstrates the effectiveness of the proposed Rao- 3 algorithm over Rao-1 and Rao-2 algorithms, as well as other algorithms. PQ bus voltage profiles obtained in Case 8 are shown in Figure 7.

Table 13. Case 8: VPI results in the IEEE 57-bus system.

\begin{tabular}{cccc}
\hline Algorithm & Fuel Cost (\$/h) & TVD (pu) & Time (s) \\
\hline Rao-3 & $42,043.2728$ & 0.5725 & 134.25 \\
\hline Rao-2 & $41,691.1102$ & 0.7645 & 136.34 \\
\hline Rao-1 & $41,688.4417$ & 0.9882 & - \\
\hline MSA [14] & $41,714.9851$ & 0.67818 & - \\
\hline MPSO [14] & $41,721.6098$ & 0.67813 & - \\
\hline MDE [14] & $41,717.3874$ & 0.6781 & - \\
\hline MFO [14] & $41,718.8659$ & 0.67796 & 75.41 \\
\hline FPA [14] & $41,726.3758$ & 0.69723 & - \\
\hline TSA [17] & $54,045.17$ & 0.72 & - \\
\hline DSA [18] & $41,699.4$ & 0.762 & - \\
\hline IMFO [38] & $41,692.7178$ & 0.7182 & - \\
\hline MFO [38] & $41,719.8471$ & 0.7551 & - \\
\hline GA [38] & $41,700.4162$ & 0.8051 & -
\end{tabular}


Table 13. Cont.

\begin{tabular}{cccc}
\hline Algorithm & Fuel Cost (\$/h) & TVD (pu) & Time (s) \\
\hline ECHT-DE [44] & $41,694.82$ & 0.81659 & - \\
\hline SF-DE [44] & $41,697.52$ & 0.77572 & - \\
\hline SP-DE [44] & $41,697.50$ & 0.77253 & 203.6 \\
\hline MGOA [45] & $41,697.9735$ & 0.7381 & - \\
\hline GOA [45] & $41,715.1396$ & 0.8260 & - \\
\hline
\end{tabular}

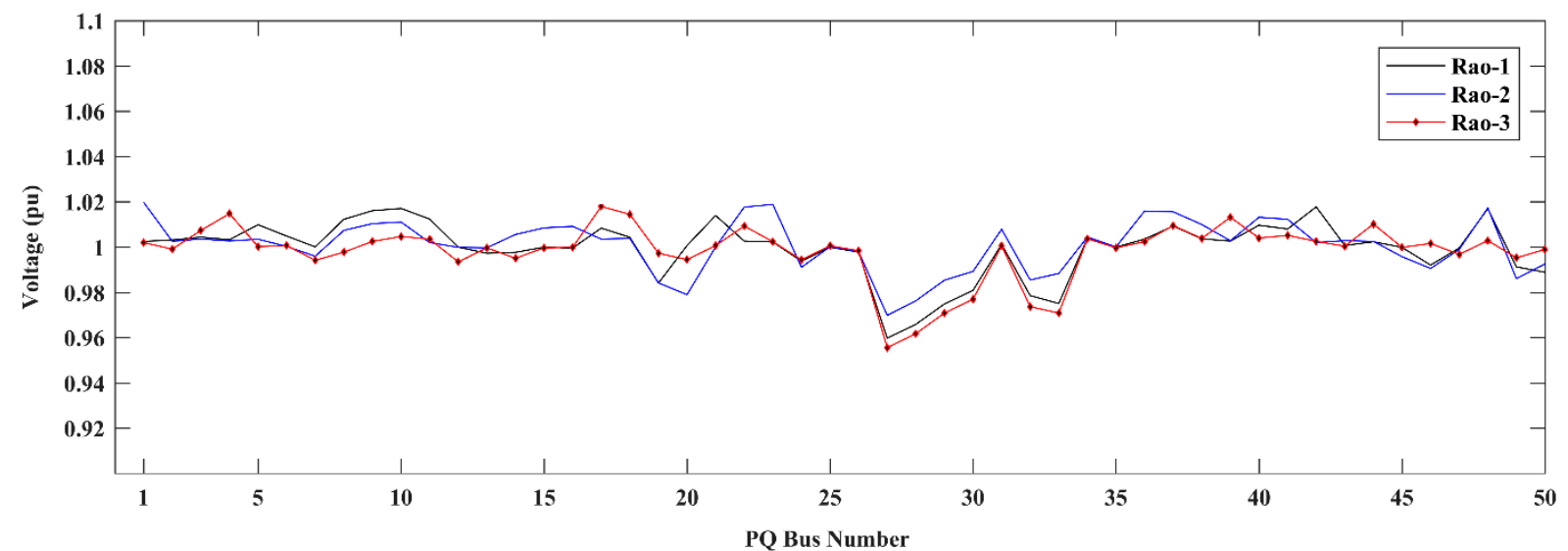

Figure 7. Voltage profile for the IEEE 57-bus system.

\subsubsection{Case 9: Voltage Stability Enhancement (VSE)}

In Case 9, the VSE is considered in addition to the fuel cost, as defined in Equation (23). The control variables' values obtained using the three Rao algorithms are provided in Table 14. The results of Case 9 attained by the proposed Rao methods and the optimization algorithms mentioned in the recent literature are compared in Table 15. The OPF results shown in Table 15 also prove the dominance of the Rao-3 algorithm over other optimization algorithms for this case.

Table 14. Optimum values of control variables of Case 9 and Case 10 for the IEEE 57-bus test system.

\begin{tabular}{|c|c|c|c|c|c|c|c|}
\hline \multirow[t]{2}{*}{ S. Number } & \multirow{2}{*}{$\begin{array}{c}\text { Control Variable } \\
\text { (p.u) }\end{array}$} & \multicolumn{3}{|c|}{$\begin{array}{l}\text { Case } 9 \\
\text { (VSE) }\end{array}$} & \multicolumn{3}{|c|}{$\begin{array}{l}\text { Case } 10 \\
\text { (RPLM) }\end{array}$} \\
\hline & & Rao-1 & Rao-2 & Rao-3 & Rao-1 & Rao-2 & Rao-3 \\
\hline \multicolumn{8}{|c|}{ Generator active power output } \\
\hline 1 & $P_{\mathrm{G} 2}$ & 0.8747 & 0.9749 & 0.9637 & 0.3048 & 0.3 & 0.3 \\
\hline 2 & $P_{\mathrm{G} 3}$ & 0.4513 & 0.4486 & 0.4518 & 1.3241 & 1.322 & 1.3549 \\
\hline 3 & $P_{\mathrm{G} 6}$ & 0.7085 & 0.7029 & 0.7067 & 0.9937 & 0.9999 & 0.9996 \\
\hline 4 & $P_{\mathrm{G} 8}$ & 4.6141 & 4.5994 & 4.5916 & 3.1132 & 3.0842 & 3.0604 \\
\hline 5 & $P_{\mathrm{G} 9}$ & 0.9998 & 0.9324 & 0.934 & 0.9978 & 0.9999 & 0.99998 \\
\hline 6 & $P_{\mathrm{G} 12}$ & 3.5892 & 3.572 & 3.5912 & 4.1 & 4.1 & 4.0999 \\
\hline \multicolumn{8}{|c|}{ Generator voltage } \\
\hline 7 & $V_{\mathrm{G} 1}$ & 1.047 & 1.0873 & 1.0873 & 1.0542 & 1.0723 & 1.0712 \\
\hline 8 & $V_{\mathrm{G} 2}$ & 1.0502 & 1.1 & 1.1 & 1.0559 & 1.0722 & 1.0721 \\
\hline 9 & $V_{\mathrm{G} 3}$ & 1.0425 & 1.0672 & 1.0676 & 1.0563 & 1.067 & 1.0666 \\
\hline
\end{tabular}


Table 14. Cont.

\begin{tabular}{|c|c|c|c|c|c|c|c|}
\hline \multirow[t]{2}{*}{ S. Number } & \multirow{2}{*}{$\begin{array}{c}\text { Control Variable } \\
\text { (p.u) }\end{array}$} & \multicolumn{3}{|c|}{$\begin{array}{l}\text { Case } 9 \\
\text { (VSE) }\end{array}$} & \multicolumn{3}{|c|}{$\begin{array}{l}\text { Case } 10 \\
\text { (RPLM) }\end{array}$} \\
\hline & & Rao-1 & Rao-2 & Rao-3 & Rao-1 & Rao-2 & Rao-3 \\
\hline 10 & $V_{\mathrm{G} 6}$ & 1.0569 & 1.0621 & 1.062 & 1.0623 & 1.0633 & 1.0631 \\
\hline 11 & $V_{\mathrm{G} 8}$ & 1.0717 & 1.0689 & 1.0697 & 1.0654 & 1.0697 & 1.0695 \\
\hline 12 & $V_{\mathrm{G} 9}$ & 1.0465 & 1.0507 & 1.0509 & 1.0492 & 1.0576 & 1.0572 \\
\hline 13 & $V_{\mathrm{G} 12}$ & 1.0328 & 1.0384 & 1.0382 & 1.0441 & 1.0572 & 1.0567 \\
\hline \multicolumn{8}{|c|}{ Tap settings } \\
\hline 14 & $T_{4-18}$ & 1.0999 & 0.9016 & 0.9 & 1.1 & 0.9117 & 1.1 \\
\hline 15 & $T_{4-18}$ & 0.9081 & 1.1 & 1.0428 & 1.0023 & 1.082 & 0.9014 \\
\hline 16 & $T_{21-20}$ & 1.0138 & 1.0321 & 1.0025 & 0.998 & 1.042 & 1.0128 \\
\hline 17 & $T_{24-25}$ & 1.083 & 1.1 & 1.0999 & 0.9036 & 1.0356 & 1.0902 \\
\hline 18 & $T_{24-25}$ & 1.1 & 1.0998 & 1.1 & 1.0698 & 0.9704 & 0.9354 \\
\hline 19 & $T_{24-26}$ & 1.0252 & 1.0268 & 1.0262 & 1.0086 & 1.0109 & 1.0098 \\
\hline 20 & $T_{7-29}$ & 0.9991 & 0.9993 & 0.9986 & 0.9953 & 0.9963 & 0.9961 \\
\hline 21 & $T_{34-32}$ & 0.9423 & 0.9522 & 0.9476 & 0.9318 & 0.9528 & 0.9526 \\
\hline 22 & $T_{11-41}$ & 0.9112 & 0.9129 & 0.9002 & 0.9111 & 0.9174 & 0.9025 \\
\hline 23 & $T_{15-45}$ & 0.9707 & 0.9909 & 0.9916 & 0.9733 & 0.9892 & 0.9889 \\
\hline 24 & $T_{14-46}$ & 0.9539 & 0.9695 & 0.9686 & 0.9671 & 0.9751 & 0.9722 \\
\hline 25 & $T_{10-51}$ & 0.9676 & 0.9709 & 0.9699 & 0.9797 & 0.9821 & 0.9819 \\
\hline 26 & $T_{13-49}$ & 0.9076 & 0.9389 & 0.9365 & 0.9411 & 0.9449 & 0.9451 \\
\hline 27 & $T_{11-43}$ & 0.9643 & 0.9782 & 0.9811 & 0.9776 & 0.9817 & 0.9939 \\
\hline 28 & $T_{40-56}$ & 0.9945 & 0.9924 & 1.0152 & 0.9826 & 0.9938 & 0.993 \\
\hline 29 & $T_{39-57}$ & 0.9753 & 0.9655 & 0.9639 & 0.96 & 0.9624 & 0.9638 \\
\hline 30 & $T_{9-55}$ & 0.9912 & 0.9934 & 0.9978 & 0.9986 & 0.9961 & 0.9947 \\
\hline \multicolumn{8}{|c|}{ Shunt VAR source } \\
\hline 31 & $Q_{\text {sh18 }}$ & 0.1139 & 0.0375 & 0.0002 & 0.2771 & 0.0002 & 0.0239 \\
\hline 32 & $Q_{\text {sh25 }}$ & 0.2412 & 0.2613 & 0.2576 & 0.1081 & 0.1448 & 0.1554 \\
\hline 33 & $Q_{\text {sh53 }}$ & 0.1405 & 0.1428 & 0.1293 & 0.1445 & 0.1339 & 0.129 \\
\hline \multicolumn{2}{|c|}{ Fuel cost $(\$ \backslash h)$} & $41,670.4726$ & $41,692.9720$ & $41,692.6149$ & $44,418.4740$ & $44,438.1623$ & $44,600.2741$ \\
\hline \multicolumn{2}{|c|}{ TVD (pu) } & 1.7637 & 1.7835 & 1.8735 & 1.5278 & 1.7464 & 1.793 \\
\hline \multicolumn{2}{|c|}{$P_{\text {Loss }}(\mathrm{MW})$} & 15.0175 & 15.561 & 15.4768 & 10.005 & 9.766 & 9.759 \\
\hline \multicolumn{2}{|c|}{ L-index } & 0.22 & 0.2191 & 0.2186 & 0.2434 & 0.2353 & 0.2330 \\
\hline
\end{tabular}

Table 15. Case 9: VSE results in the IEEE 57-bus system.

\begin{tabular}{cccc}
\hline Algorithm & Fuel Cost $\mathbf{( \$ \mathbf { h } )}$ & L-Index & Time (s) \\
\hline Rao-3 & $41,692.6149$ & 0.2186 & 131.78 \\
\hline Rao-2 & $41,692.9720$ & 0.2191 & 132.54 \\
\hline Rao-1 & $41,670.4726$ & 0.2200 & 132.76 \\
\hline MSA [14] & $41,675.9948$ & 0.27481 & - \\
\hline MPSO [14] & $41,694.1407$ & 0.27918 & - \\
\hline
\end{tabular}


Table 15. Cont.

\begin{tabular}{cccc}
\hline Algorithm & Fuel Cost (\$/h) & L-Index & Time (s) \\
\hline MDE [14] & $41,689.5878$ & 0.27677 & - \\
\hline MFO [14] & $41,680.1937$ & 0.27467 & - \\
\hline FPA [14] & $41,684.1859$ & 0.27429 & - \\
\hline DSA [18] & $41,761.22$ & 0.2383 & - \\
\hline IMFO [38] & $41,673.6204$ & 0.23525 & - \\
\hline MFO [38] & $41,688.6522$ & 0.2395 & - \\
\hline GA [38] & $41,670.0872$ & 0.2413 & - \\
\hline PSO [38] & $41,670.1755$ & 0.242 & - \\
\hline TLBO [38] & $41,685.353$ & 0.24787 & 214.4 \\
\hline SKH [43] & $43,937.1058$ & 0.2721 & - \\
\hline ECHT-DE [44] & $41,671.09$ & 0.28152 & - \\
\hline SF-DE [44] & $41,667.53$ & 0.28022 & - \\
\hline SP-DE [44] & $41,668.45$ & 0.28092 & -
\end{tabular}

\subsubsection{Case 10: Real Power Loss Minimization (RPLM)}

The function $F_{\text {Loss }}$ is selected for the RPLM as described in Equation (24). The minimum power loss attained by the Rao-2 algorithm is $9.759 \mathrm{MW}$, while Rao- 1 and Rao-3 algorithms provided the power losses of $10.005 \mathrm{MW}$ and $9.770 \mathrm{MW}$, respectively. Results of the proposed Rao algorithms and optimal control variable settings are presented in Table 14. Table 16 compares the simulation results of this case as obtained by the proposed Rao algorithms and other methods reported in recent literature. The OPF results shown in Table 16 demonstrate the superiority of the Rao-2 algorithm over Rao- 1 and Rao-3, as well as the other competitors. The power loss convergence characteristic of Case 10 is presented in Figure 8.

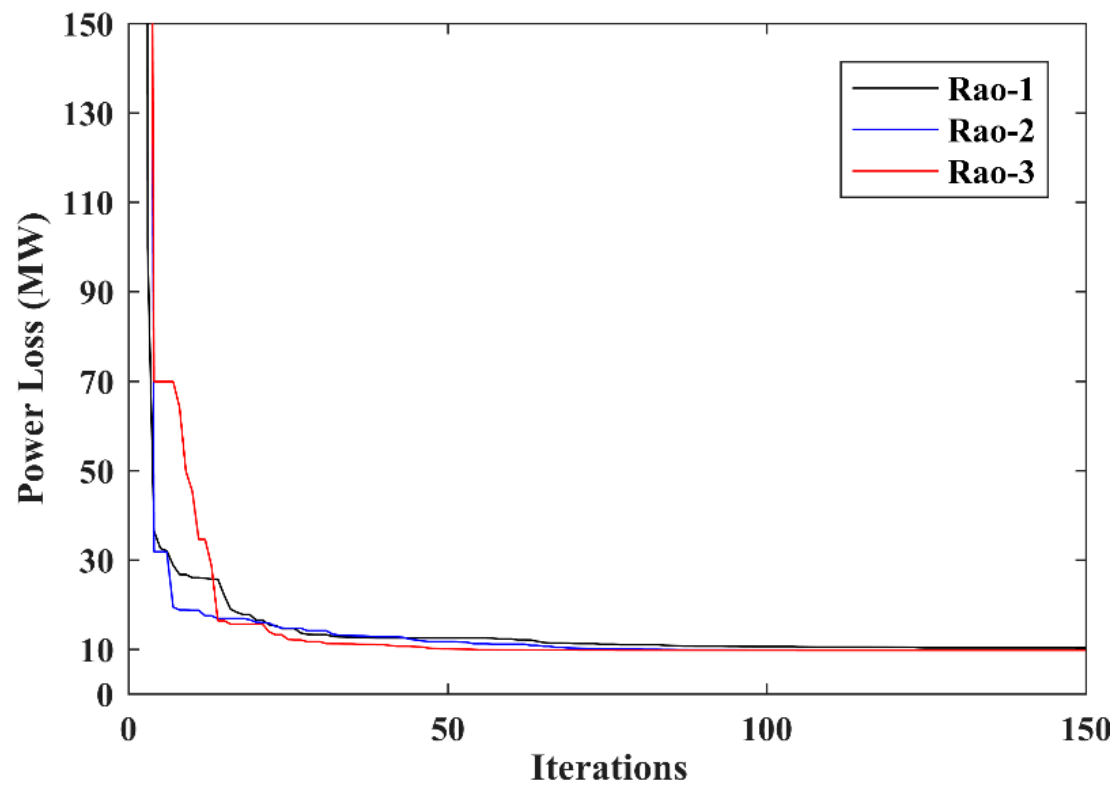

Figure 8. Convergence characteristic for the IEEE 57-bus system, Case 10. 
Table 16. Case 10: RPLM results in the IEEE 57-bus system.

\begin{tabular}{ccc}
\hline Algorithm & Power Loss (MW) & Time (s) \\
\hline Rao-3 & 9.7590 & 131.26 \\
\hline Rao-2 & 9.7660 & 132.18 \\
\hline Rao-1 & 10.005 & 135.77 \\
\hline TSA [17] & 12.473 & 76.17 \\
\hline SSA [19] & 11.321 & 81.17 \\
\hline SKH [34] & 10.6877 & - \\
\hline MSO [37] & 12.7435 & - \\
\hline CKHA [49] & 11.1224 & - \\
\hline
\end{tabular}

\subsection{IEEE 118-Bus System}

To demonstrate the scalability of Rao algorithms and prove their efficacy to solve large-scale problems, all three versions of Rao algorithms were applied to solve the OPF problem in the IEEE 118-bus test system. The IEEE 118-bus test system has 54 generation units, two reactors, and 12 capacitors, 186 branches, and nine tap-changing transformers. The system data along with the control variable operating limits are given in Reference [48]. Thirty independent runs were taken using Rao algorithms to solve the OPF problem for the proposed test system and the best results obtained out of 30 trials are shown in this section.

\section{Case 11: Fuel Cost Minimization (FCM)}

In Case 11, fuel cost is selected as the primary objective as in Case 1 . The minimum cost attained by the Rao-3 algorithm is $129,220.6794 \$ / \mathrm{h}$, while Rao-2 and Rao-1 algorithms offered the minimum fuel costs of $129,256.5242 \$ / \mathrm{h}$ and $129,241.1787 \$ / \mathrm{h}$, respectively. The OPF result of the proposed Rao-3 algorithm and the optimal control variable settings are presented in Table 17. Table 18 compares the OPF results of Case 11 obtained by the proposed Rao algorithms and other methods reported in recent articles. Based on the OPF outcomes, it is clear that the Rao-3 algorithm provided the least fuel cost as compared to the other methods. This demonstrates the effectiveness of the proposed Rao-3 algorithm over Rao-1 and Rao-2 algorithms, as well as other algorithms. The fuel cost characteristics of Case 11 is presented in Figure 9.

Table 17. Optimum values of control variables of Case 11 for the IEEE 118-bus test system.

\begin{tabular}{|c|c|c|c|c|c|c|c|c|c|c|c|}
\hline $\begin{array}{c}\text { S. } \\
\text { Number }\end{array}$ & $\begin{array}{c}\text { Control } \\
\text { Variables }\end{array}$ & Initial & Rao-3 & $\begin{array}{c}\text { S. } \\
\text { Number }\end{array}$ & $\begin{array}{c}\text { Control } \\
\text { Variables }\end{array}$ & Initial & Rao-3 & $\begin{array}{c}\text { S. } \\
\text { Number }\end{array}$ & $\begin{array}{c}\text { Control } \\
\text { Variables }\end{array}$ & Initial & Rao-3 \\
\hline 1 & $P_{\mathrm{G} 1}$ & 0 & 0.07872 & 45 & $P_{\mathrm{G} 103}$ & 0.4 & 0.33181 & 89 & $\mathrm{~V}_{\mathrm{G} 77}$ & 1.006 & 1.00706 \\
\hline 2 & $P_{\mathrm{G} 4}$ & 0 & 0.07685 & 46 & $P_{\mathrm{G} 104}$ & 0 & 0.24175 & 90 & $\mathrm{~V}_{\mathrm{G} 80}$ & 1.04 & 1.02122 \\
\hline 3 & $P_{\mathrm{G} 6}$ & 0 & 0.0662 & 47 & $P_{\mathrm{G} 105}$ & 0 & 0.06467 & 91 & $\mathrm{~V}_{\mathrm{G} 85}$ & 0.985 & 1.03344 \\
\hline 4 & $P_{\mathrm{G} 8}$ & 0 & 0.1337 & 48 & $P_{\mathrm{G} 107}$ & 0 & 0.0083 & 92 & $\mathrm{~V}_{\mathrm{G} 87}$ & 1.015 & 1.01663 \\
\hline 5 & $P_{\mathrm{G} 10}$ & 4.5 & 4.27188 & 49 & $P_{\mathrm{G} 110}$ & 0 & 0.03809 & 93 & $\mathrm{~V}_{\mathrm{G} 89}$ & 1.005 & 1.02186 \\
\hline 6 & $P_{\mathrm{G} 12}$ & 0.85 & 0.58259 & 50 & $P_{G 111}$ & 0.36 & 0.33608 & 94 & $\mathrm{~V}_{\mathrm{G} 90}$ & 0.985 & 0.9779 \\
\hline 7 & $P_{\mathrm{G} 15}$ & 0 & 0.02101 & 51 & $P_{\mathrm{G} 112}$ & 0 & 0.18375 & 95 & $\mathrm{~V}_{\mathrm{G} 91}$ & 0.98 & 0.96364 \\
\hline 8 & $P_{\mathrm{G} 18}$ & 0 & 0.00159 & 52 & $P_{\mathrm{G} 113}$ & 0 & 0.00423 & 96 & $\mathrm{~V}_{\mathrm{G} 92}$ & 0.99 & 1.0142 \\
\hline 9 & $P_{\mathrm{G} 19}$ & 0 & 0.00782 & 53 & $P_{\mathrm{G} 116}$ & 0 & 0.0008 & 97 & $\mathrm{~V}_{\mathrm{G} 99}$ & 1.01 & 0.98147 \\
\hline 10 & $P_{\mathrm{G} 24}$ & 0 & 0.0178 & 54 & $\mathrm{~V}_{\mathrm{G} 1}$ & 0.995 & 1.02416 & 98 & $\mathrm{~V}_{\mathrm{G} 100}$ & 1.017 & 1.03575 \\
\hline 11 & $P_{\mathrm{G} 25}$ & 2.2 & 2.1492 & 55 & $\mathrm{~V}_{\mathrm{G} 4}$ & 0.998 & 1.03241 & 99 & $\mathrm{~V}_{\mathrm{G} 103}$ & 1.01 & 1.05908 \\
\hline 12 & $P_{\mathrm{G} 26}$ & 3.14 & 2.84427 & 56 & $\mathrm{~V}_{\mathrm{G} 6}$ & 0.99 & 1.03808 & 100 & $\mathrm{~V}_{\mathrm{G} 104}$ & 0.971 & 1.05774 \\
\hline
\end{tabular}


Table 17. Cont.

\begin{tabular}{|c|c|c|c|c|c|c|c|c|c|c|c|}
\hline $\begin{array}{c}\text { S. } \\
\text { Number }\end{array}$ & $\begin{array}{c}\text { Control } \\
\text { Variables }\end{array}$ & Initial & Rao-3 & $\begin{array}{c}\text { S. } \\
\text { Number }\end{array}$ & $\begin{array}{c}\text { Control } \\
\text { Variables }\end{array}$ & Initial & Rao-3 & $\begin{array}{c}\text { S. } \\
\text { Number }\end{array}$ & $\begin{array}{c}\text { Control } \\
\text { Variables }\end{array}$ & Initial & Rao-3 \\
\hline 13 & $P_{\mathrm{G} 27}$ & 0 & 0.31058 & 57 & $\mathrm{~V}_{\mathrm{G} 8}$ & 1.015 & 0.97062 & 101 & $\mathrm{~V}_{\mathrm{G} 105}$ & 0.965 & 1.05895 \\
\hline 14 & $P_{\mathrm{G} 31}$ & 0.07 & 0.09645 & 58 & $\mathrm{~V}_{\mathrm{G} 10}$ & 1.05 & 0.9431 & 102 & $\mathrm{~V}_{\mathrm{G} 107}$ & 0.952 & 1.05859 \\
\hline 15 & $P_{\mathrm{G} 32}$ & 0 & 0.00434 & 59 & $\mathrm{~V}_{\mathrm{G} 12}$ & 0.99 & 1.05326 & 103 & $\mathrm{~V}_{\mathrm{G} 110}$ & 0.973 & 0.96628 \\
\hline 16 & $P_{\mathrm{G} 34}$ & 0 & 0.17751 & 60 & $\mathrm{~V}_{\mathrm{G} 15}$ & 0.97 & 1.01875 & 104 & $\mathrm{~V}_{\mathrm{G} 111}$ & 0.98 & 0.95313 \\
\hline 17 & $P_{\mathrm{G} 36}$ & 0 & 0.32141 & 61 & $\mathrm{~V}_{\mathrm{G} 18}$ & 0.973 & 1.0552 & 105 & $\mathrm{~V}_{\mathrm{G} 112}$ & 0.975 & 0.94652 \\
\hline 18 & $P_{\mathrm{G} 40}$ & 0 & 0.46268 & 62 & $\mathrm{~V}_{\mathrm{G} 19}$ & 0.962 & 1.02694 & 106 & $\mathrm{~V}_{\mathrm{G} 113}$ & 0.993 & 1.03236 \\
\hline 19 & $P_{\mathrm{G} 42}$ & 0 & 0.68702 & 63 & $\mathrm{~V}_{\mathrm{G} 24}$ & 0.992 & 1.04181 & 107 & $\mathrm{~V}_{\mathrm{G} 116}$ & 1.005 & 0.97069 \\
\hline 20 & $P_{\mathrm{G} 46}$ & 0.19 & 0.25232 & 64 & $\mathrm{~V}_{\mathrm{G} 25}$ & 1.05 & 1.04186 & 108 & $\mathrm{~T}_{5-8}$ & 0.985 & 0.9002 \\
\hline 21 & $P_{\mathrm{G} 49}$ & 2.04 & 1.87987 & 65 & $\mathrm{~V}_{\mathrm{G} 26}$ & 1.015 & 0.95537 & 109 & $\mathrm{~T}_{26-25}$ & 0.96 & 1.03159 \\
\hline 22 & $P_{\mathrm{G} 54}$ & 0.48 & 0.30277 & 66 & $\mathrm{~V}_{\mathrm{G} 27}$ & 0.968 & 1.03944 & 110 & $\mathrm{~T}_{30-17}$ & 0.96 & 0.9885 \\
\hline 23 & $P_{\mathrm{G} 55}$ & 0 & 0.74996 & 67 & $\mathrm{~V}_{\mathrm{G} 31}$ & 0.967 & 1.03063 & 111 & $\mathrm{~T}_{38-37}$ & 0.935 & 0.95581 \\
\hline 24 & $P_{\mathrm{G} 56}$ & 0 & 0.35764 & 68 & $\mathrm{~V}_{\mathrm{G} 32}$ & 0.963 & 1.02981 & 112 & $\mathrm{~T}_{63-59}$ & 0.96 & 1.09927 \\
\hline 25 & $P_{\mathrm{G} 59}$ & 1.55 & 1.53703 & 69 & $\mathrm{~V}_{\mathrm{G} 34}$ & 0.984 & 1.02045 & 113 & $\mathrm{~T}_{64-61}$ & 0.985 & 0.91596 \\
\hline 26 & $P_{\mathrm{G} 61}$ & 1.6 & 1.65333 & 70 & $\mathrm{~V}_{\mathrm{G} 36}$ & 0.98 & 1.03052 & 114 & $\mathrm{~T}_{65-66}$ & 0.935 & 1.08267 \\
\hline 27 & $P_{\mathrm{G} 62}$ & 0 & 0.09242 & 71 & $\mathrm{~V}_{\mathrm{G} 40}$ & 0.97 & 0.98601 & 115 & $\mathrm{~T}_{68-69}$ & 0.935 & 0.90129 \\
\hline 28 & $P_{\mathrm{G} 65}$ & 3.91 & 3.98068 & 72 & $\mathrm{~V}_{\mathrm{G} 42}$ & 0.985 & 0.97303 & 116 & $\mathrm{~T}_{81-80}$ & 0.935 & 1.0949 \\
\hline 29 & $P_{\mathrm{G} 66}$ & 3.92 & 3.33319 & 73 & $\mathrm{~V}_{\mathrm{G} 46}$ & 1.005 & 1.03671 & 117 & $\mathrm{Q}_{\mathrm{Sh} 5}$ & 0 & 0.0397 \\
\hline 30 & $P_{\mathrm{G} 70}$ & 0 & 0.12369 & 74 & $\mathrm{~V}_{\mathrm{G} 49}$ & 1.025 & 1.00562 & 118 & $\mathrm{Q}_{\mathrm{sh} 34}$ & 0 & 0.13264 \\
\hline 31 & $P_{\mathrm{G} 72}$ & 0 & 0.04355 & 75 & $\mathrm{~V}_{\mathrm{G} 54}$ & 0.955 & 0.96514 & 119 & $\mathrm{Q}_{\mathrm{sh} 37}$ & 0 & 0.29284 \\
\hline 32 & $P_{\mathrm{G} 73}$ & 0 & 0.01357 & 76 & $\mathrm{~V}_{\mathrm{G} 55}$ & 0.952 & 0.98114 & 120 & $\mathrm{Q}_{\mathrm{sh} 44}$ & 0 & 0.22743 \\
\hline 33 & $P_{\mathrm{G} 74}$ & 0 & 0.05 & 77 & $\mathrm{~V}_{\mathrm{G} 56}$ & 0.954 & 0.96645 & 121 & $\mathrm{Q}_{\text {sh45 }}$ & 0 & 0.16846 \\
\hline 34 & $P_{\mathrm{G} 76}$ & 0 & 0.05541 & 78 & $\mathrm{~V}_{\mathrm{G} 59}$ & 0.985 & 1.04398 & 122 & $\mathrm{Q}_{\text {sh46 }}$ & 0 & 0.01255 \\
\hline 35 & $P_{\mathrm{G} 77}$ & 0 & 0.05941 & 79 & $\mathrm{~V}_{\mathrm{G} 61}$ & 0.995 & 1.05939 & 123 & $\mathrm{Q}_{\mathrm{sh} 48}$ & 0 & 0.00779 \\
\hline 36 & $P_{\mathrm{G} 80}$ & 4.77 & 3.5775 & 80 & $\mathrm{~V}_{\mathrm{G} 62}$ & 0.998 & 1.05898 & 124 & $\mathrm{Q}_{\text {sh74 }}$ & 0 & 0.27942 \\
\hline 37 & $P_{\mathrm{G} 85}$ & 0 & 0.12274 & 81 & $\mathrm{~V}_{\mathrm{G} 65}$ & 1.005 & 0.96444 & 125 & $\mathrm{Q}_{\text {sh79 }}$ & 0 & 0.01159 \\
\hline 38 & $P_{\mathrm{G} 87}$ & 0.04 & 0.01293 & 82 & $\mathrm{~V}_{\mathrm{G} 66}$ & 1.05 & 1.05809 & 126 & $\mathrm{Q}_{\text {sh82 }}$ & 0 & 0.3 \\
\hline 39 & $P_{\mathrm{G} 89}$ & 6.07 & 4.56372 & 83 & $\mathrm{~V}_{\mathrm{G} 69}$ & 1.035 & 1.04551 & 127 & $\mathrm{Q}_{\mathrm{sh} 83}$ & 0 & 0.15625 \\
\hline 40 & $P_{\mathrm{G} 90}$ & 0 & 0.06063 & 84 & $\mathrm{~V}_{\mathrm{G} 70}$ & 0.984 & 0.94113 & 128 & $Q_{\text {sh105 }}$ & 0 & 0.13484 \\
\hline 41 & $P_{\mathrm{G} 91}$ & 0 & 0.01788 & 85 & $\mathrm{~V}_{\mathrm{G} 72}$ & 0.98 & 0.94008 & 129 & $\mathrm{Q}_{\mathrm{sh} 107}$ & 0 & 0.26686 \\
\hline 42 & $P_{\mathrm{G} 92}$ & 0 & 0.05746 & 86 & $\mathrm{~V}_{\mathrm{G} 73}$ & 0.991 & 0.94036 & 130 & $Q_{\text {sh110 }}$ & 0 & 0.01831 \\
\hline 43 & $P_{\mathrm{G} 99}$ & 0 & 0.00446 & 87 & $\mathrm{~V}_{\mathrm{G} 74}$ & 0.958 & 1.00781 & & & & \\
\hline 44 & $P_{\mathrm{G} 100}$ & 2.52 & 2.33746 & 88 & $\mathrm{~V}_{\mathrm{G} 76}$ & 0.943 & 0.96203 & & & & \\
\hline \multicolumn{10}{|c|}{ Fuel Cost $(\$ / h)$} & $131,220.0208$ & $129,220.6794$ \\
\hline & & & & & & & & \multicolumn{2}{|c|}{ TVD (p.u) } & 1.4389 & 1.5416 \\
\hline & & & & & & & & \multicolumn{2}{|c|}{$P_{\text {Loss }}(\mathrm{MW})$} & 132.8101 & 109.1203 \\
\hline & & & & & & & & \multicolumn{2}{|c|}{$Q_{\text {Loss }}(\mathrm{MW})$} & 782.6073 & 745.9912 \\
\hline & & & & & & & & \multicolumn{2}{|c|}{$\mathrm{P}_{\mathrm{G} 69}$ (slack bus) } & 513.8101 & 471.2005 \\
\hline
\end{tabular}

Table 18. Case 11 (FCM) results in the IEEE 118-bus system.

\begin{tabular}{ccc}
\hline Algorithm & Fuel Cost $\mathbf{( \$ \mathbf { h } )}$ & Time (s) \\
\hline Rao-3 & $129,220.6794$ & 164.19 \\
\hline Rao-2 & $129,256.5242$ & 169.24 \\
\hline
\end{tabular}


Table 18. Cont.

\begin{tabular}{ccc}
\hline Algorithm & Fuel Cost $\mathbf{( \$ \mathbf { h } )}$ & Time (s) \\
\hline Rao-1 & $129,241.1787$ & 167.33 \\
\hline GPU-PSO [1] & $129,627.03$ & - \\
\hline IMFO [38] & 131.8200 & - \\
\hline PSOGSA [50] & $129,733.58$ & - \\
\hline
\end{tabular}

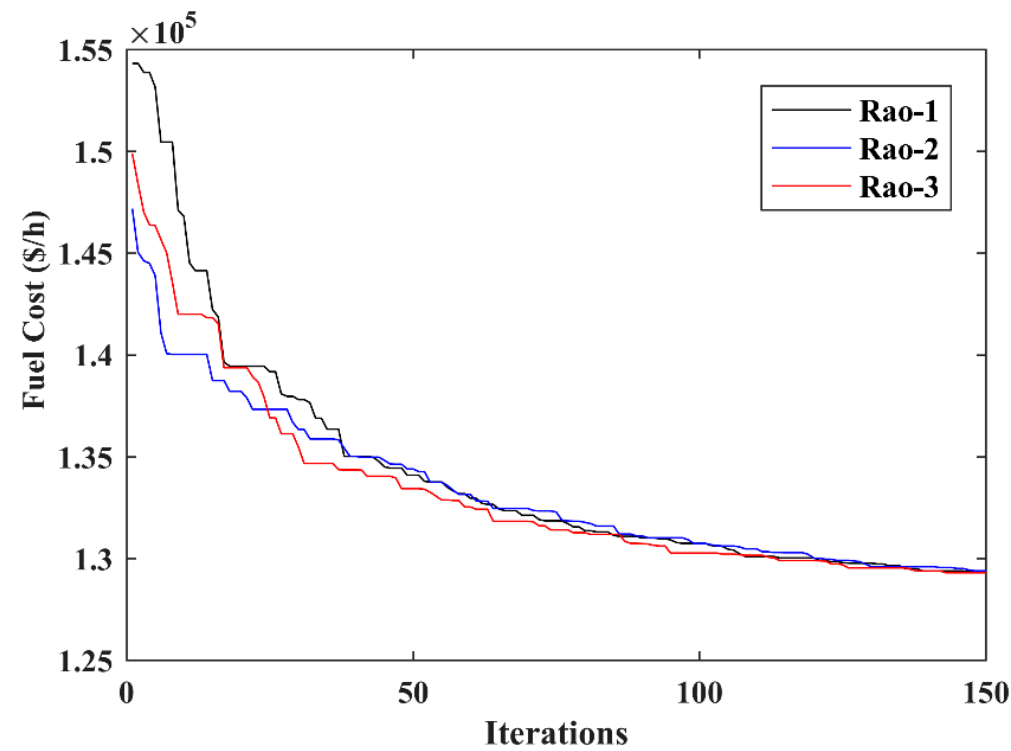

Figure 9. Fuel cost convergence characteristic of Rao algorithms for Case 11.

\section{Statistical Comparison of Rao-1, Rao-2, and Rao-3}

Table 19 presents a statistical summary of 30 independent runs performed using three variants of Rao algorithms.

Table 19. Statistical analysis of the various cases using the Rao algorithms.

\begin{tabular}{cccccccccc}
\hline Algorithm & Best & Worst & Mean & $\begin{array}{c}\text { Standard } \\
\text { Deviation }\end{array}$ & Best & Worst & Mean & $\begin{array}{c}\text { Standard } \\
\text { Deviation }\end{array}$ \\
\hline & & Case 1 & & & & & Case 7 \\
\hline Rao-3 & 799.9683 & 801.8023 & 800.8813 & 0.0186 & $41,659.2621$ & $41,674.4259$ & $41,669.0213$ & 1.7866 \\
\hline Rao-2 & 799.9918 & 801.9718 & 800.9032 & 0.0203 & $41,872.0668$ & $41,894.0668$ & $41,887.0668$ & 2.2906 \\
\hline Rao-1 & 800.4391 & 802.1403 & 801.2391 & 0.0223 & $41,771.1088$ & $41,782.4437$ & $41,776.6512$ & 2.1860 \\
\hline & & Case 5 & & & & Case 10 & 9.7971 \\
\hline Rao-3 & 3.0675 & 3.1182 & 3.0714 & 0.0288 & 9.7590 & 9.8460 & 9.0318 \\
\hline Rao-2 & 3.1086 & 3.1761 & 3.1271 & 0.0360 & 9.7660 & 9.8541 & 9.8065 & 0.0339 \\
\hline Rao-1 & 3.3041 & 3.4065 & 3.3389 & 0.0408 & 10.0050 & 10.9451 & 10.4515 & 0.0351 \\
\hline Rao-3 & 0.2126 & 0.2246 & 0.2206 & 0.0166 & $129,220.6794$ & $129,440.3458$ & $129,331.6023$ & 4.0910 \\
\hline Rao-2 & 0.2040 & 0.2065 & 0.2048 & 0.0131 & $129,256.5242$ & $129,541.2740$ & $129,402.0961$ & 4.7350 \\
\hline Rao-1 & 0.2037 & 0.2049 & 0.2043 & 0.0110 & $129,241.1787$ & $129,511.7206$ & $129,381.4028$ & 4.5210 \\
\hline
\end{tabular}




\section{Conclusions}

In this paper, newly developed algorithm-specific parameter-less optimization techniques, through Rao algorithms, are proposed to solve the OPF problem. The suggested Rao algorithms were found to be superior to other EC/SI-based methods, as these algorithms do not involve tuning the algorithm-specific parameters. Various objectives considered for solving the OPF problem in this paper included the minimization of fuel cost, minimization of total voltage deviation, enhancement of voltage stability under normal and contingency conditions, minimization of real power loss, and minimization of emission cost.

To demonstrate the efficacy of the Rao algorithms and their capacity to solve OPF problems in different scale power systems with complex constraints and large dimensions, the algorithms are applied on the standard IEEE 30-bus system with 24 control variables, the IEEE 57-bus system with 33 control variables, and the IEEE 118-bus system with 130 control variables. The simulation results achieved by the proposed Rao algorithms were compared with recently developed optimization algorithms, which proved superiority in most cases of the proposed Rao algorithms, particularly Rao-2 and Rao-3 algorithms in terms of robustness and quality of solutions. The results confirm their capability to solve complex optimization problems as well as large dimensional OPF problems. As the proposed Rao algorithms are parameter tuning-free and are capable of solving complex and large dimensional OPF problems, they can be employed to solve OPF problems in practical power systems.

Author Contributions: The presented work was developed by the following contributions: Conceptualization, methodology, S.G.; Software, formal analysis, research, writing — original draft preparation, N.K.; Writing — review and editing, and supervising, L.S.; Writing—review and editing, and supervising, H.M.; Writing — review and editing, and supervising, F.P.G.M.; Writing—review and editing, funding acquisition, A.A.-M. All authors have contributed equally in technical and non-technical work. All authors have read and agreed to the published version of the manuscript.

Funding: The work reported herein has been financially supported by the Dirección General de Universidades, Investigación e Innovación of Castilla-La Mancha under the research grant ProSeaWind project (reference: SBPLY/19/180501/000102).

Institutional Review Board Statement: Not applicable.

Informed Consent Statement: Not applicable.

Conflicts of Interest: The authors declare no conflict of interest.

\section{Nomenclature}

\begin{tabular}{|c|c|}
\hline$m$ & state variables \\
\hline$n$ & control variables \\
\hline$Z_{\text {min }}(m, n)$ & objective function \\
\hline$g(m, n)$ & equality constraint \\
\hline$h(m, n)$ & inequality constraint \\
\hline$P_{g i}$ and $Q_{g i}$ & are generator active and reactive power \\
\hline Nbus & are the set of the bus \\
\hline$N L B$ & are the set of the load bus \\
\hline$N t l$ & are the set of the transmission line \\
\hline NGN & are the set of generators units \\
\hline NC & are the set shunt compensation switch \\
\hline NTR & are the set of regulating transformers \\
\hline$P_{d i}$ and $Q_{d i}$ & are the load buses' active and reactive power demand \\
\hline$P_{\text {Loss }}$ and $Q_{\text {Loss }}$ & are the total real and reactive power loss \\
\hline$V_{g_{k}}^{\max }$ and $V_{g_{k}}^{\min }$ & are maximum and minimum bus voltage limit of the $k$ th generator bus \\
\hline$Q_{g_{k}}^{\max }$ and $Q_{g_{k}}^{\min }$ & $\begin{array}{l}\text { are the maximum and minimum limit of the reactive power output of the } k \text { th } \\
\text { generator bus }\end{array}$ \\
\hline
\end{tabular}




$P_{g_{k}}^{\text {max }}$ and $P_{g_{k}}^{\text {min }}$
$T_{k}^{\text {min }}$ and $T_{k}^{\text {max }}$
$V_{L_{k}}^{\text {min }}$ and $V_{L_{k}}^{\text {max }}$
$S_{l_{k}}^{\text {max }}$
$C_{1}, C_{2}, C_{3}$ and $C_{4}$
$A_{i}, B_{i}$, and $C_{i}$
$\delta_{i}, \mu_{i}, \gamma_{i}, \xi_{i}$ and $\lambda_{i}$
PD
NB
MPSO
MDE
MFO
FPA
QOJA
IMFO
ARCBBO
RCBBO
GWO
MGBICA
GBICA
ABC
SKH
ECHT-DE
SF-DE
SP-DE
MGOA
GOA
GPU-PSO
ALC-PSO
PSOGSA

are the maximum and minimum active power limit of the $k$ th generating units are the lower and upper voltage limit of the tap setting of the $k$ th transformer are the lower and upper voltage limit of the $k$ th load bus is the maximum MVA flow in the $k$ th branch are the penalty factors are the $i$ th generator units' fuel cost coefficients are the emission coefficients of the $i$ th generator unit active power load demand total number of buses modified particle swarm optimization modified differential evolution moth flame optimization flower pollination algorithm quasi-oppositional-based Jaya algorithm improved moth-flame optimization adaptive real-coded biogeography-based optimization real-coded biogeography-based optimization gray wolves optimization multi-objective gaussian bare-bones imperialist competitive algorithm gaussian bare-bones imperialist competitive algorithm artificial bee colony algorithm stud krill herd algorithm ensemble of constraint handling techniques-differential evolution the superiority of feasible solutions-differential evolution self-adaptive penalty-differential evolution modified grasshopper optimization algorithm grasshopper optimization algorithm graphics processing unit's particle swarm optimization particle swarm optimization with aging leader and challengers hybrid particle swarm optimization and gravitational search algorithm

\section{References}

1. Roberge, V.; Tarbouchi, M.; Okou, F. Optimal power flow based on parallel metaheuristics for graphics processing units. Electr. Power Syst. Res. 2016, 140, 344-353. [CrossRef]

2. Saha, A.; Chakraborty, A.K.; Das, P. Quasi-reaction-based symbiotic organisms search algorithm for solving static optimal power flow problem. Sci. Iran. 2019, 26, 1664-1689. [CrossRef]

3. Carpentier, J. Optimal power flows. Int. J. Electr. Power Energy Syst. 1979, 1, 3-15. [CrossRef]

4. Maskar, M.B.; Thorat, A.R.; Korachgaon, I. A review on optimal power flow problem and solution methodologies. In Proceedings of the International Conference on Data Management, Analytics and Innovation (ICDMAI), Pune, India, 24-26 February 2017; pp. 64-70. [CrossRef]

5. Momoh, J.A.; Adapa, R.; El-Hawary, M.E. A review of selected optimal power flow literature to 1993. I. Nonlinear and quadratic programming approaches. IEEE Trans. Power Syst. 1999, 14, 96-104. [CrossRef]

6. Niu, M.; Wan, C.; Xu, Z. A review on applications of heuristic optimization algorithms for optimal power flow in modern power systems. J. Mod. Power Syst. Clean Energy 2014, 2, 289-297. [CrossRef]

7. Al Rashidi, M.R.; El-Hawary, M.E. Applications of computational intelligence techniques for solving the revived optimal power flow problem. Electr. Power Syst. Res. 2009, 79, 694-702. [CrossRef]

8. Gupta, S.; Kumar, N.; Srivastava, L. Bat Search Algorithm for Solving Multi-Objective Optimal Power Flow Problem. In Applications of Computing, Automation and Wireless Systems in Electrical Engineering. Lecture Notes in Electrical Engineering; Springer: Singapore, 2019; Volume 553. [CrossRef]

9. Bouchekara, H.R.E.H.; Chaib, A.E.; Abido, M.A.; El-Sehiemy, R.A. Optimal power flow using an Improved Colliding Bodies Optimization algorithm. Appl. Soft Comput. J. 2016, 42, 119-131. [CrossRef]

10. Bouchekara, H.R.E.H. Optimal power flow using black-hole-based optimization approach. Appl. Soft Comput. J. 2014, 24, 879-888. [CrossRef]

11. Sivasubramani, S.; Swarup, K.S. Multiagent based differential evolution approach to optimal power flow. Appl. Soft Comput. J. 2012, 12, 735-740. [CrossRef]

12. Daryani, N.; Hagh, M.T.; Teimourzadeh, S. Adaptive group search optimization algorithm for multi-objective optimal power flow problem. Appl. Soft Comput. J. 2016, 38, 1012-1024. [CrossRef] 
13. Roy, P.K.; Ghoshal, S.P.; Thakur, S.S. Multi-objective Optimal Power Flow Using Biogeography-based Optimization. Electr. Power Compon. Syst. 2010, 38, 1406-1426. [CrossRef]

14. Mohamed, A.A.A.; Mohamed, Y.S.; El-Gaafary, A.A.M.; Hemeida, A.M. Optimal power flow using moth swarm algorithm. Electr Power Compon. Syst. 2017, 142, 190-206. [CrossRef]

15. Saha, A.; Das, P.; Chakraborty, A.K. Water evaporation algorithm: A new metaheuristic algorithm towards the solution of optimal power flow. Eng. Sci. Technol. Int. J. 2017, 20, 1540-1552. [CrossRef]

16. Duman, S. Symbiotic organisms search algorithm for optimal power flow problem based on valve-point effect and prohibited zones. Neural Comput. Applic. 2017, 28, 3571-3585. [CrossRef]

17. El-Fergany, A.A.; Hasanien, H.M. Tree-seed algorithm for solving optimal power flow problem in large-scale power systems incorporating validations and comparisons. Appl. Soft Comput. J. 2018, 64, 307-316. [CrossRef]

18. Abaci, K.; Yamacli, V. Differential search algorithm for solving multi-objective optimal power flow problem. Int. J. Electr. Power Energy Syst. 2016, 79, 1-10. [CrossRef]

19. El-Fergany, A.A.; Hasanien, H.M. Salp swarm optimizer to solve optimal power flow comprising voltage stability analysis. Neural Comput. Applic. 2020, 32, 5267-5283. [CrossRef]

20. Hassan, M.H.; Kamel, S.; El-Dabah, M.A.; Khurshaid, T.; Dominguez-Garcia, J.L. Optimal Reactive Power Dispatch with Time-Varying Demand and Renewable Energy Uncertainty Using Rao-3 Algorithm. IEEE Access 2021, 9, 23264-23283. [CrossRef]

21. Rao, R.V.; Keesari, H.S. Rao algorithms for multi-objective optimization of selected thermodynamic cycles. Eng. Comput. 2020, 1-29. [CrossRef]

22. Rao, R.V.; Pawar, R.B. Constrained design optimization of selected mechanical system components using Rao algorithms. Appl. Soft Comput. 2020, 89, 106141. [CrossRef]

23. Rao, R.V.; Pawar, R.B. Self-adaptive multi-population Rao algorithms for engineering design optimization. Appl. Artif. Intell. 2020, 34, 187-250. [CrossRef]

24. Premkumar, M.; Babu, T.S.; Umashankar, S.; Sowmya, R. A new metaphor-less algorithms for the photovoltaic cell parameter estimation. Optik 2020, 208, 164559. [CrossRef]

25. Sharma, S.R.; Singh, B.; Kaur, M. Classification of Parkinson disease using binary Rao optimization algorithms. Expert Syst. 2021, 38, e12674. [CrossRef]

26. Rao, R.V.; Pawar, R.B. Quasi-oppositional-based Rao algorithms for multi-objective design optimization of selected heat sinks. J. Comput. Des. Eng. 2020, 7, 830-863. [CrossRef]

27. Hassan, M.H.; Kamel, S.; Selim, A.; Khurshaid, T.; Domínguez-García, J.L. A Modified Rao-2 Algorithm for Optimal Power Flow Incorporating Renewable Energy Sources. Mathematics 2021, 9, 1532. [CrossRef]

28. Wolpert, D.H.; Macready, W.G. No free lunch theorems for optimization. IEEE Trans. Evol. Comput. 1997, 1, 67-82. [CrossRef]

29. Rao, R.V. Rao algorithms: Three metaphor-less simple algorithms for solving optimization problems. Int. J. Ind. Eng. Comput. 2020, 11, 107-130. [CrossRef]

30. Gupta, S.; Kumar, N.; Srivastava, L. Solution of optimal power flow problem using sine-cosine mutation based modified Jaya algorithm: A Case Study. Energy Sources Part A Recovery Util. Environ. Eff. 2021.

31. Gupta, S.; Kumar, N.; Srivastava, L. An efficient Jaya algorithm with Powell's Pattern Search for optimal power flow incorporating distributed generation. Energy Sources Part B Econ. Plan. Policy 2021, 1-28. [CrossRef]

32. Lee, K.Y.; Park, Y.M.; Ortiz, J.L. A United Approach to Optimal Real and Reactive Power Dispatch. IEEE Trans. Power Appar. Syst. 1985, 104, 1147-1153. [CrossRef]

33. Niknam, T.; Jabbari, M.; Reza, A. A modified shuffle frog leaping algorithm for multi-objective optimal power flow. Energy 2011, 36, 6420-6432. [CrossRef]

34. Reddy, S.S.; Bijwe, P.R.; Abhyankar, A.R. Faster evolutionary algorithm based optimal power flow using incremental variables. Int. J. Electr. Power Energy Syst. 2014, 19, 8210-8254. [CrossRef]

35. Warid, W.; Hizam, H.; Mariun, N.; Abdul-Wahab, N.I. Optimal power flow using the Jaya algorithm. Energies 2016, 9 , 678. [CrossRef]

36. Warid, W.; Hizam, H.; Mariun, N.; Wahab, N.I.A. A Novel Quasi-Oppositional Jaya Algorithm for Optimal Power Flow Solution. In Proceedings of the 2018 International Conference on Computing Sciences and Engineering (ICCSE), Kuwait City, Kuwait, 11-13 March 2018; pp. 1-5. [CrossRef]

37. Kotb, M.F.; El-Fergany, A.A. Optimal Power Flow Solution Using Moth Swarm Optimizer Considering Generating Units Prohibited Zones and Valve Ripples. J. Electr. Eng. Technol. 2020, 15, 179-192. [CrossRef]

38. Taher, M.A.; Kamel, S.; Jurado, F.; Ebeed, M. An improved moth-flame optimization algorithm for solving optimal power flow problem. Int. Trans. Electr. Energy Syst. 2019, 29, e2743. [CrossRef]

39. Kumar, R.A.; Premalatha, L. Optimal power flow for a deregulated power system using adaptive real coded biogeography-based optimization. Int. J. Electr. Power Energy Syst. 2015, 73, 393-399. [CrossRef]

40. El-Fergany, A.A.; Hasanien, H.M. Single and Multi-Objective Optimal Power Flow Using Grey Wolf Optimizer and Differential Evolution Algorithms. Electr. Power Compon. Syst. 2015, 43, 1548-1559. [CrossRef]

41. Ghasemi, M.; Ghavidel, S.; Ghanbarian, M.M.; Gitizadeh, M. Multi-objective optimal electric power planning in the power system using Gaussian bare-bones imperialist competitive algorithm. Inf. Sci. 2015, 294, 286-304. [CrossRef] 
42. Adaryani, M.R.; Karami, A. Artificial bee colony algorithm for solving multi-objective optimal power flow problem. Int. J. Electr. Power Energy Syst. 2013, 53, 219-230. [CrossRef]

43. Pulluri, H.; Naresh, R.; Sharma, V. A solution network based on stud krill herd algorithm for optimal power flow problems. Soft Comput. 2018, 22, 159-176. [CrossRef]

44. Biswas, P.P.; Suganthan, P.N.; Mallipeddi, R.; Amaratunga, G.A.J. Optimal power flow solutions using differential evolution algorithm integrated with effective constraint handling techniques. Eng. Appl. Artif. Intell. 2018, 68, 81-100. [CrossRef]

45. Taher, M.A.; Kamel, S.; Jurado, F.; Ebeed, M. Modified grasshopper optimization framework for optimal power flow solution Electr. Eng. 2019, 101, 121-148. [CrossRef]

46. Abido, M.A. Optimal power flow using tabu search algorithm. Electr. Power Compon. Syst. 2002, 30, 469-483. [CrossRef]

47. Reddy, S.S.; Bijwe, P.R. An efficient optimal power flow using bisection method. Electr. Eng. 2018, 100, 2217-2229. [CrossRef]

48. Power Systems Test Case Archive: University of Washington. Available online: http://www.ee.washington.edu/research/pstca/ (accessed on 1 August 2021).

49. Prasada, D.; Mukherjee, A.; Mukherjee, V. Application of chaotic krill herd algorithm for optimal power flow with direct current link placement problem. Chaos Solitons Fractals 2017, 103, 90-100. [CrossRef]

50. Radosavljević, J.; Klimenta, D.; Jevtić, M.; Arsić, N. Optimal Power Flow Using a Hybrid Optimization Algorithm of Particle Swarm Optimization and Gravitational Search Algorithm. Electr. Power Compon. Syst. 2015, 43, 1958-1970. [CrossRef] 